\title{
N75-20066
}

NASA CR-132613

\section{RADIATION OF CYLINDRICAL DUCT ACOUSTIC MODES WITH FLOW MISMATCH}

\author{
by \\ S. D. Savkar with I. H. Edelfelt \\ Prepared under Contract No. NAS 1-11984 \\ by \\ General Electric Corporate Research and Development \\ P. O. Box 8 \\ Schenectady, New York 12301 \\ for
}

NATIONAL AERONAUTICS AND SPACE ADMINISTRATION

March 1975 
NASA CR-132613

\section{RADIATION OF CYLINDRICAL DUCT ACOUSTIC MODES WITH FLOW MISMATCH}

by

S. D. Savkar with I. H. Edelfelt

Prepared under Contract No. NAS 1-11984

by

General Electric Corporate Research and Development

P. O. Box 8

Schenectady, New York 12301

for

NATIONAL AERONAUTICS AND SPACE ADMINISTRATION

March 1975

SRD $-75-029$ 


\section{TABLE OF CONTENTS}

$\underline{\text { PAGE }}$

SUMMARY

1

I . INTRODUCTION 2

II. THEORY FOR THE RADIATION OF SPINNING DUCT WAVE 4 GUIDE MODES

II.1 The Exhaust Mode - Formulation 4

II.2 Decomposition of the Wiener-Hopf Kernel 9

II.2.a The Carrier-Koiter Approximation 9

II.2.b Decomposition of $T *(\alpha)$ and $f_{3}(\alpha) \quad 11$

II.2.c Integral Decomposition of $f_{2}(\alpha) \quad 12$

II.2. $d$ Product Decomposition of $f_{1}(\alpha) \quad 14$

II.2.e Decomposition of $f_{4}(\alpha) \quad 17$

II.3 The Radiation Pattern and Conversion 18 Coefficients

$\begin{array}{lll}\text { II.3.a } & \begin{array}{l}\text { Asymptotic Solution to the } \\ \text { Radiation Pattern }\end{array} & 18 \\ \text { II.3.b } & \begin{array}{l}\text { Evaluation of the Conversion } \\ \text { Coefficients }\end{array} & 20\end{array}$

II.4 Influence of a Doubly Infinite Centerbody 21

II.5 The Inlet Problem 24

III. DISCUSSION OF SAMPLE CALCULATIONS 26

IV. CONCLUSIONS $\quad 30$

$\begin{array}{ll}\text { REFERENCES } & 31\end{array}$ 


\section{LIST OF ILLUSTRATIONS}

FIGURE

Asymptotic Approximation of $\Omega(x)$ Function.

The Radiation Field.

Plane Mode Reflection Coefficients.

Plane Mode Reflection Coefficients.

Phase of Modes Reflected by an Incident Plane Wave.

Comparison of Plane Wave Radiation Patterns, Data from Plumblee [6]. Calculations with Hub Tip Ratio of .566 .

11 Comparison of a Hubless Plane Wave Radiation Pattern with Data of Plumblee [6].

12 Comparison of Experimental and Calculated Reflection Coefficients.

13 Calculated Effect of Mach Number on the Plane Wave Reflection.

14 Comparison of Theoretical Asymmetric (3, 1) Radiation Pattern with Data of Plumblee [6].

15 Illustration of the Influence of Flow Mismatch and Flight Effect. Compare with Data of Plumblee [6] for the $(4,1)$ Mode.

16 Effect of Increasing Jet Issue Mach Number.

17 Trial Calcualtions for an Inlet Mode and Comparison with Crigler and Copeland [8] Fan Data for 53 Bladed Fan and 62 Bladed IGV (9 Lobe Spinning Mode).

18 Annular Inlet Directivity Patterns, Comparison of Theory with Phase Speaker Array Data of Pasko [19]. 
Physical Exhaust Jet Speed and Sound Frequency.

\section{LIST OF TABLES}

TABLE

TITLE

PAGE

I

Comparison of Calculated Plane Mode

Radiation Impedances with Experimental

Data. 
FOREWORD

The research described herein, which was conducted by the General Electric Corporate Research and Development Center, was performed under NASA Contract NAS1-11984. The work was initially done under the management of the NASA Project Manager, Mr. D. L. Lansing, and in the latter stage under Dr. W. Zorumski, Acoustics Branch, NASA Langley Research Center.

The authors acknowledge with pleasure the considerable influence of Dr. R. Mani, GE CR\&D, on this research effort. The unstinting advise of Dr. G. M. Roe, GE CR\&D, in developing the mathematical routines deserves a special mention. Useful discussions with Dr. M. Kurosaka, GE CR\&D, are gratefully acknowledged.

The complex Bessel function routine eventually employed in the subject calculations, was developed and graciously made available to us by Mr. Phipps of the Computations Branch, GE Aircraft Engine Business Group, Evendale, Ohio. 
RADIATION OF CYLINDRICAL DUCT ACOUSTIC

MODES WITH FLOW MISMATCH

by S. D. Savkar

with I. H. Edelfelt

General Electric Corporate R\&D

\section{SUMMARY}

Calculations for the radiation of spinning acoustic modes, with or without a centerbody, and with or without flow temperature and velocity discontinuity, are presented. Solutions to the appropriate convected wave equations devised around Fourier transforms and Wiener-Hopf technique are presented. The decomposition of the asymmetric kernel, resulting from a flow and temperature mismatch, is carried out in part exactly and partially using the so-called Carrier-Koiter approximation procedure. The resulting solutions offer a good approximation to the radiation of both symmetric and asymmetric modes through a flow discontinuity represented as a plug flow jet issuing from a cylindrical duct. Besides the Koiter approximation, the major limitation on the calculation program is the difficulty of calculating the high order Bessel functions with sufficient accuracy.

Sample calculations for symmetric modes are presented offering comparisons between the current calculations and in particular available experimental data such as that of Plumblee et al [6] and Mechel et al [7]. So long as one restricts attention to the amplitude functions, such as the radiation pattern or the absolute value of the conversion (reflection) coefficients, there is generally a qualitative agreement, even here there are certain peculiar features of the predictions which do not appear to be reproduced experimentally for reasons discussed. The agreement between the calculated and measured phase values in the presence of flow appear to be rather poor.

In the case of the asymmetric modes, limited comparisons between calculated and measured radiation patterns appear to be reasonable, thus in general reinforcing the conclusion regarding ability to predict amplitude functions. However none of the data available go to significantly high enough Mach number. For this one must rely purely on calculations.

The calculations suggest that the influence of the Mach number discontinuity is to offer a conflict between the effects of convection and refraction. The former becoming increasingiy more significant at high subsonic Mach numbers by the presence of a strong lobe in the shadow zone. Effects of flight conditions are to weaken such conflicts so that in the limit of uniform Mach number, one recovers Carrier's well-known solution.

The effect of a temperature mismatch is rather more dramatic, resulting in a sharp beaming of sound off to the side. This is consistent with the kinematic picture of a 
plane wave impinging on a temperature discontinuity.

I. INTRODUCTION

In their now classic paper, Tyler and Sofrin [1] demonstrated that turbomachinery noise propagates as spinning acoustic modes and is eventually radiated to the far field. While the qualitative features of the physical process were brought out, their use of a radiation field uncoupled from the process within the duct and no flow left a number of unanswered questions. There appeared to be a need for the solution of the complete radiation problem coupling the field within the duct to the far field in the manner of the Levine and Schwinger [2] paper and its extension to a duct immersed in a uniform flow by Carrier [3]. From the point of view of turbomachinery application, the need was to be able to handle high lobe number spinning (asymmetric) duct wave guide modes and the flow discontinuity present as for example in the exhaust mode. The first poses computational difficulties since it requires an ability to handle high order Bessel functions accurately. Lansing et al. [4] have developed a theory for the asymmetric modes in extension of the Carrier paper [3]. However the use of an isothermal, uniformly flowing medium excludes such features as refraction and an enhanced mismatch between the duct and the surrounding. The refractive influence of the flow mismatch was demonstrated by Mani [5], who employed a form of the Carrier-Koiter approximation scheme. Unfortunately these calculations are restricted to flat ducts.

While the theory has been slow in evolving, the experimental measurements of relevant model problems have not been easy to come by. Since the problem of primary interest must include a flowing medium, the measurements have been limited by the inadequacy of acoustic instrumentation in turbulent flow. Then there is the problem of generating suitably well defined acoustic modes, especially the asymmetric spinning modes of interest. Perhaps the most comprehensive attempt along these lines is that of Plumblee et al. [6], who have developed an annular flow duct apparatus capable of handling flow velocities up to $200 \mathrm{ft} / \mathrm{sec}$. together with the acoustic instrumentation necessary to map the acoustic fields both within the duct and in the radiation field. Plumblee et al's. [6] attempt at measuring spinning mode radiation impedances are unique. One of the very few other measurements available are those of Mechel et al. [7], who measured the plane mode reflection coefficients both with and without flow. When it comes to more realistic measurements, as from fans, the only available data are for the radiation field. Even these in general do not offer a good basis for discerning the influence of specific modes and moreover the picture is clouded over by the incoherent scattering of the noise by flow turbulence and velocity gradients. The problem is clearly more severe in the exhaust mode than the inlet mode. Crigler and Copeland [8] have made some rather detailed measurements of a fan radiation field which appears to be strongly dominated by a rotor-IGV interaction of a definitive modal character. 
In this report we shall attempt a solution to the problem of radiation of spinning duct acoustic modes of high lobe order and include in our consideration a flow mismatch both in the sense of a jet exhaust flow and a temperature mismatch between the jet fluid and the surrounding. The problem in a sense represents the fan exhaust or turbine exhaust problem. However we shall model this using a cylinder of zero wall thickness from which issues a plug flow jet through which upstream sound refracts and radiates to the far field. We shall also approximate the influence of a centerbody by allowing the inner boundary to be a doubly infinite "rod". The solution will be devised around the appropriate convected wave equation using the particle displacement matching boundary condition and the Wiener-Hopf technique. In the process of the solution, we shall resort to the Carrier-Koiter approximation scheme to render the problem of decomposing the Wiener-Hopf kernel tractable. The validity of the scheme has been extensively discussed and illustrated by both Koiter [9] and Carrier [10] . One notes also the successful use of the same by Mani [5] in the flat duct problem.

We shall attempt an evaluation of our calculation routine by comparing sample predictions with the earlier discussed and other experimental results. We expect at the outset reasonable correlation for the radiation patterns but not for the reflection coefficients, or more precisely the phase of the reflection coefficient due to difficulties resulting from experimental conditions and more importantly the presence of turbulent flow.

Finally, we shall also offer some interesting results related to temperature mismatch.

\section{NOMENCLATURE}

a radius of duct, used to nondimensionalize all the length parameters

c speed of sound

$\mathrm{H}_{\mathrm{m}}^{(1)}(\mathrm{Z})$ Hankel Function of the first kind

$I_{m}(Z) \quad$ modified Bessel Function of the first kind

j $\sqrt{-1}$

$\mathrm{J}_{\mathrm{m}}(\mathrm{z}) \quad$ Bessel Function of first kind order $\mathrm{m}$

$\mathrm{k}$

reduced frequency, wa/c

$\mathrm{K}_{\mathrm{m}}(\mathrm{Z}) \quad$ modified Bessel Function of the second kind

M Mach number

p pressure

$Y_{m}(Z) \quad$ Bessel Function of the second kind 


$\begin{array}{ll}\alpha & \text { Fourier transform variable } \\ \sigma & \text { hub to tip ratio of the centerbody } \\ \rho & \text { density } \\ \omega & \text { circular frequency } \\ \phi & \text { velocity potential } \\ \mu_{\mathrm{ms}} & \text { sth zero of the appropriate Bessel function or cross } \\ & \text { product order } \mathrm{m} \\ & \text { particle displacement }\end{array}$

II. THEORY FOR THE RADIATION OF SPINNING DUCT WAVE GUIDE MODES

\section{II.1 The Exhaust Mode - Formulation}

Consider now the problem of a monochromatic spinning acoustic mode lobe number ' $m$ ' in semi-infinite duct of radius ' $a=1$.' The duct wall is assumed to be of a negligible thickness and is located at $r=1 ., x<0$ as in Figure 1 , where $(r, \theta, x)$ represent a cylindrical coordinate frame. The analysis will be restricted to small disturbances in perfect, inviscid, nonheat conducting media. In the formal analysis, however, an allowance will be made for a mismatch between the flow within the duct and the surrounding medium. The region formed by the duct and its phantom extension will be designated as region ' $I$ ' or ' 1 ' in the subscripts. The surrounding region is then II or 2 in the subscripts. In what follows the frequency of the incident signal, propagating in the duct toward the open end, will be prescribed to be ' $\omega$ ' and the time dependence of the form $\exp (-j \omega t), j=\sqrt{-1}$, will be assumed. Then the disturbance velocity potential amplitude $\phi$ is governed by the convected form of the Helmholtz equation

$$
\nabla^{2} \phi+\left(k+j M \frac{\partial}{\partial x}\right)^{2} \phi=0
$$

where it is understood that $M=M_{1}$ in region $I$ and $M=M_{2}$ in region $I I$ and that $k=k_{1}=\omega a / c_{1}$ and $k=k_{2}=\omega a / c_{2}$ respectively. The latter makes an allowance for differencess in temperatures (or gases) between regions I and II. The solution to the above problem is to be carried out subject to the boundary conditions:

a) Along the walls of the duct the transverse acoustic particle velocity be zero, i.e.

$$
\frac{\partial \phi}{\partial r}=0 \quad r=1 \quad \text { and } x<0
$$

b) The acoustic pressure be continuous on the extension of the duct into $x>0$, 


$$
\phi_{1}+j \frac{M_{1}}{k_{1}} \frac{\partial \phi_{1}}{\partial x}=\phi_{2}+j \frac{M_{2}}{k_{2}} \frac{\partial \phi_{2}}{\partial x}, \quad r=1 .
$$

c) In addition to the above, noting the definition

$$
\frac{\mathrm{D} n}{\mathrm{Dt}}=\frac{\partial \phi}{\partial \mathrm{r}},
$$

it is required that the radial acoustic particle displacements in the two regions be matched along the streamlines separating the two regions. This is the boundary condition proposed by Ribner [12] and Miles [13]. There are some interesting questions related to this boundary condition, but we defer these to a later section.

Adopt now a representation in terms of the Fourier transforms, so that in the two regions I, II, we write, taking a circumferential dependence of the form $\exp (j \mathrm{~m} \theta)$ :

$$
\phi_{1}=\int_{-\infty}^{\infty} A_{+}(\alpha) \frac{I_{m}\left(\gamma_{1} r\right)}{\gamma_{1} I_{m}^{\prime}\left(\gamma_{1}\right)} e^{-j \alpha x} d \alpha
$$

and

$$
\phi_{2}=\int_{-\infty}^{\infty} B_{+}(\alpha) \frac{K_{m}\left(\gamma_{2} r\right)}{\gamma_{2} K_{m}^{\prime}\left(\gamma_{2}\right)} e^{-j \alpha x} d \alpha
$$

where $\alpha$ is the Fourier transform parameter $I_{m}$ and $K_{m}$ being modified Bessel functions of the 1st and 2 nd kind

and

$$
\gamma^{2}=\alpha^{2}-(k+\alpha M)^{2}
$$

It is easily verified that equations 2 and 4 and 3 and 4 satisfy the wave equation. The ' + ' signs in (2) and (3) designate functions regular and free of zeros in the upper half $\alpha$ plane, to be defined presently. In the procedure we have adopted, we shall draw heavily on the book by Noble [11]. Hence to simplify repeated references, such as to specific equations or pages, we shall adopt the notation $(N, 1.15)$ in reference to equation (1.15) of Noble or page $(\mathrm{N}-10)$ as referring to page 10 of Noble [11].

Now the function $\gamma$ of a clearly has two branch points at $k /(1-M)$ and $-k /(1+M)$, so we introduce branch cuts such that $\gamma \rightarrow+\infty$ as $\alpha \rightarrow \pm \infty$. As discussed by Noble, the cuts can be made in several different ways, however for our subsequent calculations the cuts which turn out to be convenient are illustrated in Figure 2, where for the sake of illustration it is assumed that $k$ has a small, but positive imaginary 
component, i.e.*

$$
\mathbf{k}=\mathbf{k}_{\mathbf{r}}+\mathbf{i} \varepsilon, \quad \text { taking } \quad \alpha=\xi+j \eta .
$$

The cuts are along the hyperbolas defined by $\xi \eta=\mathrm{k} \varepsilon$, in the limit $\varepsilon \rightarrow 0$ these reduce to cuts such as illustrated, for example, by Figure 3. Actually, it turns out that the cuts for $\gamma_{1}$ could just as conveniently have been made to extend horizontally from $\mathrm{k}_{1} /\left(1-M_{1}\right)$ to $+\infty$ and $-\mathrm{k}_{1} /\left(1+\mathrm{M}_{1}\right)$ to $-\infty$, but we adopt the above convention for consistency. This allows us now to define the upper and lower half planes (taking $M_{1}$, $\mathrm{M}_{2}$ to be positive) as

upper half plane - for $\operatorname{Im}(\alpha)>$ the larger of $\operatorname{Im}\left(-\mathrm{k}_{2} /(1+\right.$

$$
\left.\mathrm{M}_{2}\right) \text { ) or } \operatorname{Im}\left(-\mathrm{k}_{1} /\left(1+\mathrm{M}_{1}\right)\right)
$$

and

lower half plane - for $\operatorname{Im}(\alpha)<$ the lesser of $\operatorname{Im}\left(\mathrm{k}_{2} /(1\right.$ -

$$
\left.\mathrm{M}_{2}\right) \text { ) or } \operatorname{Im}\left(\mathrm{k}_{1} /\left(1-\mathrm{M}_{1}\right)\right)
$$

Consequently the path of integration in (1) and (2) is taken to pass between the branch cuts in the region of commonality (shaded region in Figure 2 ).

Clearly the form of solution adopted ensures the satisfaction of the boundary condition (a). Hence consider an incident wave of the form

$$
p_{\text {inc }}=J_{m}\left(\mu_{m n} r\right) \exp \left(j K_{m n} x\right)
$$

or

$$
\phi_{i n c}=\frac{-j}{\rho_{1} \omega} \frac{J_{m}\left(\mu_{m n} r\right)}{\left(1-M_{1} \bar{K}_{m n} / k_{1}\right)} \exp \left(j K_{m n} x\right)
$$

where $K_{m n}$ the axial propagation constant is defined as

*Writing $\alpha=\xi+j \eta$, the branch is defined as

$$
\gamma=|\gamma| e^{j\left(\frac{\theta_{1}+\theta_{2}}{2}\right)}
$$

with the ranges $\quad-2 \pi+\arctan \Lambda^{+}<\theta_{1}<\arctan \Lambda^{+}$ $\arctan \Lambda^{-}<\theta_{2}<\arctan \Lambda^{-}+2 \pi$

where

$$
\begin{aligned}
& \Lambda^{+}=\frac{\eta-\varepsilon /(1-M)}{\xi-\mathrm{k}_{\mathrm{r}} /(1-M)} \\
& \Lambda^{-}=\frac{\eta+\varepsilon /(1+M)}{\xi+\mathrm{k}_{\mathrm{r}} /(1+M)}
\end{aligned}
$$




$$
\mathrm{K}_{\mathrm{mn}}=\frac{\sqrt{\mathrm{k}_{1}^{2}-\left(1-\mathrm{M}_{1}^{2}\right) \mu_{m n}^{2}}-\mathrm{k}_{1} \mathrm{M}_{1}}{1-\mathrm{M}_{1}^{2}}
$$

with the branch chosen being $\operatorname{Im}\left(\mathrm{K}_{\mathrm{mn}}\right) \geq 0$, corresponding to downstream propagation, $\mu_{\text {p }}$ being the zero of $J^{\prime}\left(\mu_{m n}\right)=0$, satisfying boundary condition (a). Then the radiapnacoustic particle displacement is

or

$$
\eta=\frac{j \partial \phi / \partial r}{\omega\left(1-M K_{m n} / k\right)}
$$

$$
\eta_{i n c}=\frac{\mu_{m n} J_{m}^{\prime}\left(\mu_{m n} r\right)}{\rho_{1} \omega^{2}\left(1-M_{1} K_{m n} / k_{1}\right)^{2}} \exp \left(j K_{m n} x\right)
$$

By its definition the incident wave particle displacement vanishes as $\mathbf{r} \rightarrow 1$ for all $\mathbf{x}$. Again, the acoustic particle displacements corresponding to (2) and (3) are

$$
\begin{aligned}
& \eta_{1}=\frac{j}{\omega} \int_{-\infty}^{\infty} A_{+}(\alpha) \frac{I_{m}^{\prime}\left(\gamma_{1} r\right)}{\left(1+\alpha M_{1} / k_{1}\right) I_{m}^{\prime}\left(\gamma_{1}\right)} e^{-j \alpha x} d \alpha \\
& \eta_{2}=\frac{j}{\omega} \int_{-\infty}^{\infty} B_{+}(\alpha) \frac{K_{m}^{\prime}\left(\gamma_{2} r\right)}{\left(1+\alpha M_{2} / k_{2}\right) K_{m}^{\prime}\left(\gamma_{2}\right)} e^{-j \alpha x} d \alpha
\end{aligned}
$$

Substituting into the boundary condition (c) and noting that ${ }_{\mathrm{B}_{+}}^{\eta_{\text {inc }}} \equiv 0$ for $\mathrm{r}=1_{-}$, there obtains the relation between $\mathrm{A}_{+}$and

$$
\frac{A_{+}(\alpha)}{\left(1+\alpha M_{1} / k_{1}\right)}-\frac{B_{+}(\alpha)}{\left(1+\alpha M_{2} / k_{2}\right)}=F_{-}(\alpha)
$$

The left and the right hand sides being regular and zero free respectively in the upper and lower half planes. Noting the behavior of the two sides as $\mathrm{x} \rightarrow 0$, by the standard application of the Wiener-Hopf argument and the Liouville theorem, each side is equal to a constant taken to be zero. There obtains the relation

$$
A_{+}(\alpha)=\frac{\left(1+\alpha M_{1} / k_{1}\right)}{\left(a+\alpha M_{2} / k_{2}\right)} B_{+}(\alpha)
$$

The remaining boundary condition, continuity of acoustic pressure

$$
p_{2}\left(1_{+}\right)=p_{1}\left(1_{-}\right)+p_{\text {inc }}\left(1_{-}\right) \quad \text { for } x>0
$$

noting the definitions 


$$
\begin{aligned}
& p_{1}=j \rho_{1} \omega \int_{-\infty}^{\infty} A_{+}(\alpha) \frac{\left(1+\alpha M_{1} / k_{1}\right) I_{m}\left(\gamma_{1} r\right)}{\gamma_{1} I_{m}^{\prime}\left(\gamma_{1}\right)} e^{-j \alpha x} d \alpha \\
& p_{2}=j \rho_{2} \omega \int_{-\infty}^{\infty} B_{+}(\alpha) \frac{\left(1+\alpha M_{2} / k_{2}\right) K_{m}\left(\gamma_{2} r\right)}{\gamma_{2} K_{m}^{\prime}\left(\gamma_{2}\right)} e^{-j \alpha x} d \alpha
\end{aligned}
$$

and on $\mathbf{r}=1_{\text {_ }}$ for $\mathrm{x}>0$, writing

$$
p_{i n c}=\frac{j J_{m}\left(\mu_{m n}\right)}{2 \pi} \int_{-\infty}^{\infty} \frac{e^{-j \alpha x}}{\left(\alpha+K_{m n}\right)} d \alpha
$$

since

$$
e^{j K_{m n} x}=-\frac{1}{2 \pi j} \int_{-\infty}^{\infty} \frac{e^{-j \alpha x}}{\alpha+K_{m n}} d \alpha
$$

then yields the functional equation

$$
\begin{gathered}
\left\{\frac{\rho_{2}}{\rho_{1}} \frac{\left(1+\alpha \mathrm{M}_{2} / \mathrm{k}_{2}\right) \mathrm{K}_{\mathrm{m}}\left(\gamma_{2}\right)}{\gamma_{2} \mathrm{~K}_{\mathrm{m}}^{\prime}\left(\gamma_{2}\right)}-\frac{\left(1+\alpha \mathrm{M}_{1} / \mathrm{k}_{1}\right)^{2}}{\left(1+\alpha \mathrm{M}_{2} / \mathrm{k}_{2}\right)} \frac{\mathrm{I}_{\mathrm{m}}\left(\gamma_{1}\right)}{\gamma_{1} \mathrm{I}_{\mathrm{m}}^{\prime}\left(\gamma_{1}\right)}\right\} \times \\
\times \mathrm{B}_{+}(\alpha)-\frac{\mathrm{J}_{\mathrm{m}}\left(\mu_{\mathrm{mn}}\right)}{2 \pi \rho_{1} \omega\left(\alpha+\mathrm{K}_{\mathrm{mn}}\right)}=G_{-}(\alpha)
\end{gathered}
$$

where the equality is understood to apply to the region of commonality (shaded region of Figure 2), and the function $G_{-}(\alpha)$ is regular and free of zeros in the lower half $\alpha$ plane. Denote by $L(\alpha)$ the grouping in the curly brackets. Then equation (15) may be rewritten as

$$
L(\alpha) B_{+}(\alpha)-\frac{J_{m}\left(\mu_{m n}\right)}{2 \pi \rho_{1} \omega\left(\alpha+K_{m n}\right)}=G_{-}(\alpha)
$$

Then if the function $L(\alpha)$ can be decomposed into factors $L_{+}(\alpha)$ and $L_{-}(\alpha)$ regular and free of zeros in the upper and lower half $\bar{p}$ lanes

$$
L(\alpha)=L_{+}(\alpha) L_{-}(\alpha)
$$

The standard application of the Wiener-Hopf arguments and the Liouville theorem yields

$$
B_{+}(\alpha)=\frac{J_{m}\left(\mu_{m n}\right)}{2 \pi \rho_{1} \omega\left(\alpha+\bar{K}_{m n}\right) L_{+}(\alpha) L_{-}\left(-K_{m n}\right)}
$$

Except for the decomposition of $L(\alpha)$, this completes the 
formal solution to the probiem posed.

\section{II.2 Decomposition of the Wiener-Hopf Kerne1}

\section{II.2.a The Carrier-Koiter Approximation}

Recall the definition of the kernel function $L(\alpha)$ :

$$
L(\alpha)=\frac{\rho_{2}}{\rho_{1}} \frac{\left(1+\alpha \mathrm{M}_{2} / \mathrm{k}_{2}\right) \mathrm{K}_{\mathrm{m}}\left(\gamma_{2}\right)}{\gamma_{2} K_{m}^{\prime}\left(\gamma_{2}\right)}-\frac{\left(1+\alpha \mathrm{M}_{1} / \mathrm{k}_{1}\right)^{2}}{\left(1+\alpha \mathrm{M}_{2} / \mathrm{k}_{2}\right)} \frac{\mathrm{I}_{\mathrm{m}}\left(\gamma_{1}\right)}{\gamma_{1} \mathrm{I}_{\mathrm{m}}^{\prime}\left(\gamma_{1}\right)}
$$

Formally speaking the required split can be carried as per theorem $c$, page $(\mathrm{N}-15)$, of Noble, whence

$$
\ln \left(L_{+}(\alpha)\right)=\frac{1}{2 \pi j} \int_{-\infty}^{\infty} \frac{\ln (L(\zeta))}{\zeta-\alpha} d \zeta
$$

with the integration along the lower boundary of the crosshatched region in Figure 2

and

$$
\ln \left(L_{-}(\alpha)\right)=\frac{-1}{2 \pi j} \int_{-\infty+j r}^{\infty+j r} \frac{\ln (L(\zeta))}{\zeta-\alpha} d \zeta
$$

the integration path being along the upper boundary of the crosshatched region of Figure 2. However, it does not appear possible to carry out the integrations explicitly, or reduce them to simpler integrals as would be the case if $L(\alpha)$ were purely an even function of $\alpha$. In view of this, the decomposition was carried out using the Carrier-Koiter approximation scheme $[9,10]$. The applicability of this approximation to the acoustic radiation from a flat duct has been previously demonstrated by Mani [5]. To motivate the approximation, regroup $L(\alpha)$ as follows.

$$
L(\alpha)=\frac{\left(1+\alpha M_{1} / k_{1}\right)^{2}}{\left(1+\alpha M_{2} / k_{2}\right)} \frac{2}{\gamma_{1} I_{m}^{\prime}\left(\gamma_{1}\right) \gamma_{2} K_{m}^{\prime}\left(\gamma_{2}\right)}\left[\frac{e^{\left(\gamma_{1}-\gamma_{2}\right)}}{4} T(\alpha)\right]
$$

where

$$
\begin{gathered}
T(\alpha)=2 e^{\left(\gamma_{2}-\gamma_{1}\right)} \gamma_{2} I_{m}\left(\gamma_{1}\right) K_{m}^{\prime}\left(\gamma_{2}\right) H(\alpha) \\
H(\alpha)= \\
\frac{\rho_{2}}{\rho_{1}} \frac{\left(1+\alpha M_{2} / k_{2}\right)^{2}}{\left(1+\alpha M_{1} / k_{1}\right)^{2}} \frac{\gamma_{1}}{\gamma_{2}} \times \\
\\
\left.\frac{K_{m}\left(\gamma_{2}\right) I_{m}^{\prime}\left(\gamma_{1}\right)}{I_{m}\left(\gamma_{1}\right) K_{m}^{\prime}\left(\gamma_{2}\right)}-1\right\}
\end{gathered}
$$


We will presently need the limit lim $H(\alpha)$, which is readily shown to be $\alpha \rightarrow \infty$

$$
\lim _{\alpha \rightarrow \infty} H(\alpha) \rightarrow-\left[\frac{\Gamma_{2}}{\Gamma_{1}}\left(\frac{M_{2}}{M_{1}}\right)^{2} \sqrt{\frac{1-M_{1}^{2}}{1-M_{2}^{2}}}+1\right]
$$

assuming the same static pressure within and without the jet, $\rho_{1} c_{1}^{2}=\rho_{2} c_{2}^{2}$ and where $\Gamma_{2}$ and $\Gamma_{1}$ are the ratio's of specific heats in reglons II and $I^{2}$ respectively. In the case of uniform flow, $M_{1}=M_{2}$ and $\rho_{2} / \rho_{1}=1$, the square bracketed quantity in (19a) is essentially a normalized Wronskian. Consider the substitute kernel $L^{*}(\alpha)$ defined as

$L^{*}(\alpha)=\frac{\left(1+\alpha M_{1} / k_{1}\right)^{2}}{\left(1+\alpha M_{2} / k_{2}\right)} \frac{2}{\gamma_{1} I_{m}^{\prime}\left(\gamma_{1}\right) \gamma_{2} K_{m}^{\prime}\left(\gamma_{2}\right)}\left[e^{\left(\gamma_{1}-\gamma_{2}\right)} T^{*(\alpha)}\right]$ (20a) with $T^{*}(\alpha)$ being defined as the quotient approximation

$$
\mathrm{T} *(\alpha)=\frac{\delta \beta}{\lambda} \frac{\alpha^{2}+\alpha+\delta / \beta}{\alpha^{2}+\alpha+\delta / \lambda}
$$

With $\frac{\delta \beta}{\lambda}=\lim _{\alpha \rightarrow \infty}\left[-\sqrt{\gamma_{2} / \gamma_{1}} H(\alpha)\right]$ it is clear that $L^{*}(\alpha) \rightarrow \mathrm{L}(\alpha)$ in the limit as $\alpha \rightarrow \infty$ along the real axis. A more complete match according to the Carrier-Koiter rules is then obtained by matching $L(\alpha)$ and $L^{*}(\alpha)$ at $\alpha=0$ up to its first moment:

$$
\begin{aligned}
& \delta=T(0) \\
& \delta / \lambda=\frac{T(\infty)-T(0)}{T^{\prime}(0)} \\
& \delta / \beta=(\delta / \lambda) \frac{T(0)}{T(\infty)}
\end{aligned}
$$

The objective now is to carry out the decomposition of $L^{*}(\alpha)$. To facilitate the subsequent discussion, group $L^{*}(\alpha)$ as

$$
L *(\alpha)=\frac{1}{8} \frac{f_{3}(\alpha) f_{4}(\alpha)}{f_{1}(\alpha) f_{2}(\alpha)} T^{*}(\alpha)
$$

where $T^{*}(\alpha)$ is defined by equation (20b) and the functions $f_{i}$ are defined as

$$
\begin{aligned}
& f_{1}(\alpha)=\left(\frac{\gamma_{1}}{2}\right)^{-m+1} e^{-\gamma_{1}} I_{m}^{\prime}\left(\gamma_{1}\right) \\
& f_{2}(\alpha)=\left(\frac{\gamma_{2}}{2}\right)^{m+1} e^{\gamma_{2}} K_{m}^{\prime}\left(\gamma_{2}\right) \\
& f_{3}(\alpha)=\left(1+\alpha M_{1} / k_{1}\right)^{2} /\left(1+\alpha M_{2} / k_{2}\right)
\end{aligned}
$$




$$
f_{4}(\alpha)=\left(\frac{\gamma_{2}}{\gamma_{1}}\right)^{m}
$$

In terms of this simplified notation, the integral decomposition of $L^{*}(\alpha)$ is readily carried out. For an example, $L_{+}^{*}(\alpha)$ is simply expressed as

$$
\begin{aligned}
& \ln \left[L_{+}^{*}(\alpha)\right]=\frac{1}{2 \pi j}\left\{\int_{-\infty+j s}^{\infty} \frac{\ln [1 / 8 \mathrm{~T} *(\zeta)]}{\zeta-\alpha} d \zeta\right. \\
& +\int_{-\infty+j s}^{+j s} \frac{\ln \left[f_{3}(\zeta)\right]}{\zeta-\alpha} d \zeta \\
& +\int_{-\infty}^{\infty}+j s \frac{\ln \left[f_{4}(\zeta)\right]}{\zeta-\alpha} d \zeta \\
& -\int_{-\infty+j s}^{\infty} \frac{\ln f_{1}(\zeta)}{\zeta-\alpha} d \zeta \\
& \left.-\int_{-\infty+j s}^{\infty+j s} \frac{\ln \left[f_{2}(\zeta)\right]}{\zeta-\alpha} d \zeta\right\}
\end{aligned}
$$

One can similarly write down the factor $L^{*}(\alpha)$. Each integral will now be treated separately in the following subsections.

II.2.b Decomposition of $T *(\alpha)$ and $f_{3}(\alpha)$

The decomposition of the quadratic quotient $\mathrm{T} *(\alpha)$ is trivial, the roots of the quadratic terms being

$$
\begin{aligned}
& \mathrm{R}_{1,2}=-\frac{1}{2} \pm \sqrt{1 / 4-\delta / \beta} \\
& \mathrm{R}_{3,4}=-\frac{1}{2} \pm \sqrt{1 / 4-\delta / \lambda}
\end{aligned}
$$

Quotient groupings such as for example 


$$
\sqrt{\delta \beta / \lambda} \frac{\alpha-R_{1}}{\alpha-R_{3}} \quad \text { or } \quad \sqrt{\delta \beta / \lambda} \frac{\alpha-R_{1}}{\alpha-R_{4}} \quad \text { etc. }
$$

being assigned to the appropriate half planes by testing the location of the roots $R_{i}$.

The decomposition of the remainder kernel can be carried out exactly. Since the factor $\left(1+\alpha \mathrm{M}_{1} / \mathrm{k}_{1}\right)^{2} /\left(1+\alpha \mathrm{M}_{2} / \mathrm{k}_{2}\right)$ or $f_{3}(\alpha)$ is clearly a plus function, hence write

$$
\begin{aligned}
& f_{3}^{+}(\alpha)=f_{3}(\alpha) \\
& f_{3}^{-}(\alpha)=1
\end{aligned}
$$

II.2.c Integral Decomposition of $\mathrm{f}_{2}(\alpha)$

The grouping $f_{2}(\alpha)$ was motivated by the suggestion made by Noble (page $N-130$ ). It should be noticed that $f_{2}(\alpha)$ is in fact a function exclusively of $\gamma_{2}$, hence we may write

$$
f_{2}(\alpha)=f\left(\gamma_{2}\right)=\left(\frac{\gamma_{2}}{2}\right)^{m+1} e^{\gamma_{2}} K_{m}^{\prime}\left(\gamma_{2}\right)
$$

The function $f\left(\gamma_{2}\right)$ is free of zeros in the entire complex $\alpha$ plane. Integral decomposition of this function can be further simplified by making the following transformations in the $\alpha$ plane

$$
\begin{aligned}
& \tilde{\alpha}_{02}=\alpha\left(1-M_{2}^{2}\right)^{\frac{1}{2}}-\frac{k_{2} M_{2}}{\left(1-M_{2}^{2}\right)^{\frac{1}{2}}} \\
& \tilde{k}_{2}=k_{2} /\left(1-M_{2}^{2}\right)^{\frac{1}{2}}
\end{aligned}
$$

in terms of the $\tilde{\alpha}_{02}$ plane, $f\left(\gamma_{2}\right)$ is an even function of $\tilde{\alpha}_{02}$. This at once allows us to simplify the integrations involved by using the procedure ' $c$ ' of Noble, page (N-19). Hence deform the contour of integration in the $\tilde{\alpha}_{02}$ plane as per Figure 3 , then

$$
\begin{aligned}
& f_{2}^{+}=f_{+}=\left\{f\left(\gamma_{2}\right)\right\}^{\frac{1}{2}} e^{\frac{+g\left(\tilde{\alpha}_{02}\right)}{2}} \\
& f_{2}^{-}=f_{-}=\left\{f\left(\gamma_{2}\right)\right\}^{\frac{1}{2}} e^{\frac{-g\left(\tilde{\alpha}_{02}\right)}{2}}
\end{aligned}
$$


We omit here the details of the algebra involved in deducing an expression for $g(\xi)$ with simply a reference to Noble [11] (pages $\mathrm{N}-19$ and $\mathrm{N}-20$ ). Then for $|\xi| \leq \tilde{\mathrm{k}}_{2}$, it is easily verified that

$$
\begin{aligned}
& g(\xi)=\frac{2 \xi}{\pi} \mathrm{P} \int_{0}^{\tilde{\mathrm{k}}_{2}} \frac{\Omega(\mathrm{u}) \mathrm{u} \mathrm{du}}{\left\{\left(\tilde{\mathrm{k}}_{2}^{2}-\xi^{2}\right)-\mathrm{u}^{2}\right\} \sqrt{\tilde{\mathrm{k}}_{2}^{2}-\mathrm{u}^{2}}} \\
& -\frac{2 \xi}{\pi} \mathrm{P} \int_{0}^{\tilde{\mathrm{k}}_{2}} \frac{\mathrm{u}^{2} \mathrm{du}}{\left\{\left(\tilde{\mathrm{k}}_{2}^{2}-\xi^{2}\right)-\mathrm{u}^{2}\right\} \sqrt{\tilde{\mathrm{k}}_{2}^{2}-\mathrm{u}^{2}}} \\
& +\frac{2 j \xi}{\pi} \int_{0}^{\infty} \frac{\Omega\left(\sqrt{\mathrm{k}_{2}^{2}+\mathrm{y}^{2}}\right)}{\mathrm{y}^{2}+\xi^{2}} d y \\
& -\frac{2 j \xi}{\pi} \int_{0}^{\infty} \frac{\sqrt{\mathrm{k}_{2}^{2}+\mathrm{y}^{2}}}{\mathrm{y}^{2}+\xi^{2}} d y \\
& -\frac{j \xi}{|\xi|} \Omega\left(\sqrt{\mathrm{k}_{2}^{2}-\xi^{2}}\right)+\frac{j \xi}{|\xi|} \sqrt{\mathrm{k}_{2}^{2}-\xi^{2}} \\
& \Omega(x)=\arctan \left(Y_{m}^{\prime}(x) / J_{m}^{\prime}(x)\right)-\frac{\pi}{2}
\end{aligned}
$$

with

' $P$ ' preceeding the integrals denoting that they are to be taken in the sense of principal value. The integrations can be considerably simplified and carried out explicitly for $\widetilde{\mathrm{k}}_{2}$ sufficiently greater than the first zero $\mu_{m 1}$ of $J_{m}^{\prime}(x)=0$, by using the asymptotic expansions of $\Omega(\mathrm{x})$ given by Abramovitz and Stegun [14], thus

$$
\Omega(x) \approx x-\left(\frac{m}{2}+\frac{1}{4}\right)+\frac{4 m^{2}+3}{8 x}+\frac{16 m^{4}+184 m^{2}-63+\ldots}{384 x^{3}}
$$

Usefulness of the above expansions is well illustrated by the . sample comparisons for $\mathrm{m}=Q$ and $\mathrm{m}=10$ in Figures 4 and 5 . It appears to suffice that $k_{2}$ be more than roughly halfway between $\mu_{m 1}$ and $\mu_{m 2}$. Hence obtain for $0 \leq \xi<\tilde{\mathrm{k}}_{2}$ and $\tilde{\mathrm{k}}_{2}$ sufficiently $>\mu_{m 1}$, using only the first 3 tērms of the above expansion

$$
g(\xi)=\frac{-2 \xi}{\pi \tilde{k}_{2}} \int_{0}^{\frac{\pi}{2}} \frac{\sin \theta \Omega\left(\tilde{k}_{2} \sin \theta\right)-\sqrt{1-\xi^{2} / \tilde{k}_{2}^{2}} \Omega\left(\sqrt{\mathrm{k}_{2}^{2}-\xi^{2}}\right)}{\left(\sin ^{2} \theta+\xi^{2} / \tilde{k}_{2}^{2}-1\right)} d \theta
$$




$$
+\xi-j \frac{2}{\pi} \frac{4 \mathrm{~m}^{2}+3}{8 \sqrt{\tilde{\mathrm{k}}_{2}^{2}-\xi^{2}}}\left[\frac{\pi}{2}-\cos ^{-1} \frac{\xi}{\tilde{\mathrm{k}}_{2}}\right]
$$

Provided $\tilde{\mathrm{k}}_{2}$ is sufficiently larger than $\mu_{\mathrm{m} 1}$, one may readily deduce other similar formulae. We shall present here only two additional formulae of interest in deducing the conversion coefficients in mismatched flow, thus first for $\xi>\widetilde{\mathrm{k}}_{2}>\mu_{\mathrm{m} 1}$. we have

$$
\begin{aligned}
g(\xi) & =-\frac{2 \xi}{\pi} \int_{0}^{\tilde{\mathrm{k}}_{2}} \frac{\Omega(\mathrm{x}) \mathrm{x}-\tilde{\mathrm{k}}_{2}^{2}+\xi^{2}}{\left\{\left(\mathrm{x}^{2}-\tilde{\mathrm{k}}_{2}^{2}+\xi^{2}\right) \sqrt{\tilde{\mathrm{k}}_{2}^{2}-\mathrm{x}^{2}}\right.} \mathrm{dx}+\xi \\
& -\frac{2 j}{\pi}\left[\left(\frac{\mathrm{m}}{2}+\frac{1}{4}\right) \frac{\pi^{2}}{2}-\frac{4 \mathrm{~m}^{2}+3}{8 \sqrt{\xi^{2}-\tilde{\mathrm{k}}_{2}^{2}}} \ln \left(\frac{\xi}{\tilde{\mathrm{k}}_{2}}+\sqrt{\xi^{2} / \tilde{\mathrm{k}}_{2}^{2}-1}\right)\right]
\end{aligned}
$$

and for $\xi_{\tilde{\mathrm{k}}}^{2}=\tilde{\mathrm{k}}_{2}^{2}>\mu_{\mathrm{m}, 1}^{2}$

$$
\begin{aligned}
g(\xi) & =\frac{2 \xi}{\pi} \int_{0}^{\tilde{k}} \frac{\Omega(x) x-x^{2}}{\left\{\left(\tilde{k}_{2}^{2}-\xi^{2}\right)-x^{2}\right\} \sqrt{k_{2}^{2}-x^{2}}} d x \\
& +j\left[-\left(\frac{m}{2}+\frac{1}{4}\right) \pi+\frac{2 \xi}{\pi} \frac{4 m^{2}+3}{8 \tilde{k}_{2}^{2}}\right]
\end{aligned}
$$

II.2.d Product Decomposition of $f_{1}(\alpha)$

$$
\begin{aligned}
& \text { Consider next the factor } f_{1}(\alpha) \\
& \qquad f_{1}(\alpha)=\frac{e^{-\gamma_{1}} I_{m}^{\prime}\left(\gamma_{1}\right)}{\left(\gamma_{1} / 2\right)^{m-1}}
\end{aligned}
$$

The factor $e^{-\gamma_{1}}$ can in fact be extracted and treated separately. Using the branch cuts illustrated schematically in Figures 2, 3 , the decomposition of $e^{-\gamma} 1$ can be carried out in a manner parallel to the procedure used for $f_{2}(\alpha)$. Since the results are identical, for the sake of brevity, we quote instead results adopted from Noble ( $\mathrm{N}-1.35$ ) making the transformation

$$
\begin{aligned}
& \tilde{\alpha}_{01}=\alpha\left(1-M_{1}^{2}\right)^{\frac{1}{2}}-\frac{k_{1} M_{1}}{\left(1-M_{1}^{2}\right)^{\frac{1}{2}}} \\
& \tilde{k}_{1}=k_{1} /\left(1-M_{1}^{2}\right)^{\frac{1}{2}}
\end{aligned}
$$


Thus the exponential function regular and free of zeros in the upper half plane for $0 \leq \tilde{\alpha}_{01} \leq \tilde{\mathbf{k}}_{1}$ is

$$
\exp \left\{-\frac{\gamma_{1}}{\pi} \operatorname{arc} \cos \left(\tilde{\alpha}_{01} / \tilde{k}_{1}\right)\right\}
$$

with its analytic continuation for $\tilde{\alpha}_{01}>\tilde{\mathbf{k}}_{1}$

$$
\exp \left\{-j \frac{\gamma_{1}}{\pi} \ln \left(\frac{\tilde{\alpha}_{01}+\gamma_{1}}{\tilde{\mathbf{k}}_{1}}\right)\right\}
$$

so that in the limit $\tilde{\alpha}_{01} \rightarrow \infty$ in the upper half plane, it behaves as

$$
\exp \left\{-j \frac{\tilde{\alpha}_{01}}{\pi} \ln \left(2 \tilde{\alpha}_{01} / \tilde{k}_{1}\right)\right\}
$$

There now remains the factor $I_{m}^{\prime}\left(\gamma_{1}\right) /\left(\gamma_{1} / 2\right)^{m-1}$. Denote by $f_{1}(\alpha)$ its inverse

$$
\tilde{f}_{1}(\alpha)=\frac{\left(\gamma_{1} / 2\right)^{m}-1}{\bar{I}_{m}^{\prime}\left(\gamma_{1}\right)} \rightarrow \frac{\left(\tilde{\gamma}_{1} / 2\right)^{m}-1}{J_{m}^{\prime}\left(\tilde{\gamma}_{1}\right)}
$$

recalling that $\gamma_{1}=-j \tilde{\gamma}_{1}$ for $-k_{1} /\left(\frac{1}{x_{1}}+M_{1}\right)<\alpha<k_{1} /\left(1-M_{1}\right)$. In this form the decomposition of $\widetilde{f}_{1}(\alpha){ }_{\text {is }}$ readity accomplished using the infinite product form of $J_{j}^{\prime}\left(\tilde{\gamma}_{1}\right)$. We shall again omit the details of this well documented procedure with reference to Noble, pages $(\mathrm{N}-128)$ and $(\mathrm{N}-129)$, and simply note the results:

$$
\tilde{f}_{1}^{+}(\alpha)=\sqrt{\frac{2\left(\tilde{\gamma}_{1} / 2\right)^{m}}{\tilde{\gamma}_{1} J_{m}^{\prime}\left(\tilde{\gamma}_{1}\right)} s_{=}^{N}{ }^{N} \frac{\hat{\alpha}_{1, s}^{-}-\alpha}{\hat{\alpha}_{1, s}^{+}+\alpha}} e^{\frac{\tilde{\alpha}_{01}}{2}+j Y_{1}(\alpha)}
$$

where

$$
\begin{aligned}
& \hat{\alpha}_{1, s}^{+}=\frac{\sqrt{k_{1}^{2}-\mu_{m, s}^{2}\left(1-M_{1}^{2}\right)}-k_{1} M_{1}}{\left(1-M_{1}^{2}\right)} \\
& \hat{\alpha}_{1, \quad s}^{-}=\frac{\sqrt{k_{1}^{2}-\mu_{m, s}^{2}\left(1-M_{1}^{2}\right)}+k_{1} M_{1}}{\left(1-M_{1}^{2}\right)}
\end{aligned}
$$

$N$ being the highest number for which $\hat{\alpha}_{1}, s$ are real, and 


$$
\begin{aligned}
& \mathrm{Y}_{1}(\alpha)=-\frac{\tilde{\alpha}_{01}}{\pi}\left[1-c+\ln \frac{2 \pi}{\tilde{\mathrm{k}}_{1}}+\sum_{\mathrm{s}}^{\infty} \stackrel{\infty}{=}\left(\frac{1}{\mathrm{~s}}-\frac{\pi}{\mu_{\mathrm{m}, \mathrm{s}}}\right)\right] \\
& -\sum_{s}^{\infty} \frac{\tilde{\alpha}_{1}}{\mu_{\mathrm{m}, \mathrm{s}}}+\sum_{\mathrm{s}}^{\infty} \arcsin \frac{\tilde{\alpha}_{01}}{\left(\sigma_{1, \mathrm{~s}}^{2}+\tilde{\alpha}_{01}^{2}\right)^{\frac{1}{2}}}
\end{aligned}
$$

with

$$
\sigma_{1, s}^{2}=\mu_{m, s}^{2}-\tilde{k}_{1}^{2}
$$

and

$$
\mathcal{C}=\text { Euler constant }=0.5772 \ldots
$$
One can write down a similar expression for $\tilde{f}_{1}^{-}(\alpha)$ or simply
note that

$$
\tilde{\mathbf{f}}_{1}(\alpha)=\tilde{\mathrm{f}}_{1}^{+}(\alpha) \tilde{\mathrm{f}}_{1}^{-}(\alpha)
$$

The function $\mathrm{Y}_{1}(\alpha)$ can be put into a form more convenient for computation by expanding the arc sin into a series and using the Digamma function, $\psi(Z)$. Thus, $\Gamma(Z)$ here being the Gamma function,

$$
\psi(Z)=\Gamma^{\prime}(Z) / \Gamma(Z)
$$

and

$$
\sum_{s}^{\infty} 1^{\frac{1}{s}-\frac{1}{s+z}=c+\psi(1+z)}
$$

find

$$
\begin{aligned}
& Y_{1}(\alpha)=-\frac{\tilde{\alpha}_{01}}{\pi}\left[1+\psi\left(N+\frac{m}{2}+\frac{1}{4}\right)+\ln 2 \pi / \tilde{k}_{1}\right] \\
& -\frac{\tilde{\alpha}_{01}}{\pi} \quad \sum_{s}^{\infty} \frac{1}{s+N+\frac{m}{2}-\frac{3}{4}}-\frac{\pi}{\sqrt{\sigma_{1, s}^{2}+N+\tilde{\alpha}_{01}^{2}}}
\end{aligned}
$$

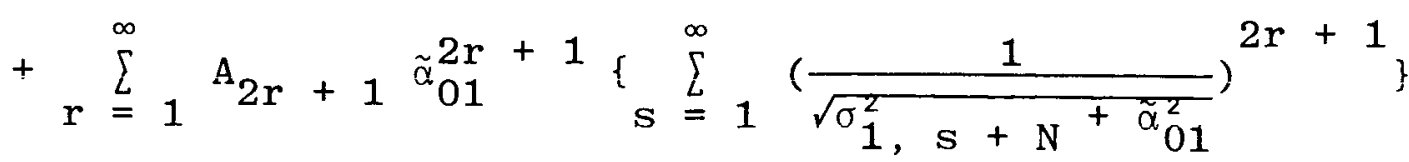

$$
\begin{aligned}
& \text { with } \quad A_{2 r}+1=\left[\int_{s=1}^{r}(2 s-1)\right] /\left[2^{r} r !(2 r+1)\right]
\end{aligned}
$$

The choice of the particular form of the grouping now becomes 
clear. Since in the limit $\alpha \rightarrow \infty$ in the upper half plane, for example, the factor regular and free of zeros in the upper half tends to (Noble page $(\mathrm{N}-128)$ ).

$$
\begin{aligned}
f_{1}^{+}(\alpha)= & {\left[\frac{e^{-\gamma_{1}} I_{m}^{\prime}\left(\gamma_{1}\right)}{\left(\gamma_{1} / 2\right)^{m}-1}\right]^{+} \rightarrow } \\
& \exp \left[-\left\{x(\alpha)-\frac{\tilde{\alpha}_{01}}{j \pi} \ln \left(\frac{2 \tilde{\alpha}_{01}}{\tilde{k}_{1}}\right)\right.\right. \\
- & \frac{\tilde{\alpha}_{01}}{j \pi}(1-c)+\left(\frac{\tilde{\alpha}_{01}}{j \pi}+\frac{m}{2}-\frac{3}{4}+\frac{1}{2}\right) \ln \left(\frac{\tilde{\alpha}_{01}}{j \pi}+\frac{m}{2}+\frac{1}{4}\right) \\
& \left.\left.\quad-\frac{\tilde{\alpha}_{01}}{j \pi} \sum_{s} \stackrel{\infty}{=}\left(\frac{1}{s}-\frac{\pi}{\mu_{m}, s}\right)\right\}\right]
\end{aligned}
$$

Hence together with the remainder of the kernel, the trailing edge condition is satisfied by picking $\chi(\alpha)$ to be

$$
\begin{aligned}
x(\alpha)= & \frac{\tilde{\alpha}_{01}}{2}-\frac{j \tilde{\alpha}_{01}}{\pi}\left[1-c+\ln \left(\frac{2 \pi}{\tilde{k}_{1}}\right)\right. \\
& \left.+\sum_{s} \stackrel{\infty}{=}\left(\frac{1}{s}-\frac{\pi}{\mu_{m, s}}\right)\right]
\end{aligned}
$$

II.2.e Decomposition of $\mathrm{f}_{4}(\alpha)$

This now leaves just one factor to be decomposed $f_{f}(\alpha)=$ $\left(\gamma_{2} / 2\right)^{\mathrm{m}} /\left(\gamma_{1} / 2\right)^{\mathrm{m}}$ which was introducted in conjunction with the earlier factors.

$$
\mathrm{f}_{4}(\alpha)=\left(\frac{\gamma_{2}}{\gamma_{1}}\right)^{\mathrm{m}}
$$

and note that $\frac{f_{4}^{\prime}(\alpha)}{f_{4}(\alpha)}=m\left(\frac{\gamma_{2}^{\prime}}{\gamma_{2}}-\frac{\gamma_{1}^{\prime}}{\gamma_{1}}\right)$

Hence obtain the factor $\mathrm{f}_{4}^{+}(\alpha)$ closing the contour in the upper half plane 


$$
\mathrm{f}_{4}^{+}(\alpha)=\left(\frac{\tilde{\gamma}_{2}}{\tilde{\gamma}_{1}}\right)^{\frac{m}{2}}\left[\frac{\tilde{\mathrm{k}}_{2}+\tilde{\alpha}_{02}}{\tilde{\mathrm{k}}_{2}-\tilde{\alpha}_{02}} \cdot \frac{\tilde{\mathrm{k}}_{1}-\tilde{\alpha}_{01}}{\tilde{\mathrm{k}}_{1}+\tilde{\alpha}_{01}}\right]^{\frac{\mathrm{m}}{2}}
$$

with $f_{4}^{-}(\alpha)=f_{4}(\alpha) / f_{4}^{+}(\alpha)$.

This completes the discussion of the Wiener-Hopf decomposition of $L^{*}(\alpha)$ into two factors $L_{*}(\alpha)$ and $L_{*}^{*}(\alpha)$. These now allow us to readily deduce quantities of physical interest.

\section{II.3 The Radiation Pattern and Conversion Coefficients}

II.3.a Asymptotic Solution to the Radiation Pattern

The simplest procedure by which to deduce the radiation pattern is to asymptotically evaluate the integral pressure expression in the limit $\left(\mathrm{r}^{2}+\mathrm{x}^{2}\right)^{\frac{1}{2}} \rightarrow \infty$. The expression for the acoustic pressure field in region II obtains from (13) and (18)

$$
\begin{gathered}
\mathrm{p}_{2}=j \rho_{2} \omega \int_{-\infty}^{\infty} \frac{\mathrm{J}_{\mathrm{m}}\left(\mu_{\mathrm{mn}}\right)\left(1+\alpha \mathrm{M}_{2} / \mathrm{k}_{2}\right)}{2 \pi \rho_{1} \omega\left(\alpha+\mathrm{K}_{\mathrm{mn}}\right) \mathrm{L}_{+}^{*}(\alpha) L_{-}^{*}\left(-\mathrm{K}_{\mathrm{mn}}\right)} \times \\
\frac{\mathrm{K}_{\mathrm{m}}\left(\gamma_{2} \mathrm{r}\right)}{\gamma_{2} \mathrm{~K}_{\mathrm{m}}^{\prime}\left(\gamma_{2}\right)} e^{-j \alpha \mathrm{x}} \mathrm{d} \alpha
\end{gathered}
$$

It is convenient now to use the spherical coordinate frame $(\mathrm{R}, \mathbb{1} \mathrm{A}, \Phi)$ centered at the duct termination, then with reference to Figure 6

$$
\begin{aligned}
& \mathrm{x}=\mathrm{R} \cos \mathbb{A} \\
& r=R \sin (1)
\end{aligned}
$$

Thus in the limit $\mathrm{R} \rightarrow \infty$ (or more precisely $\mathrm{R} \sin \mathrm{A} \rightarrow \infty$ ) one can use the asymptotic expression for $\mathrm{K}_{\mathrm{m}}\left(\gamma_{2} \mathrm{R} \sin (\mathbb{A})\right.$

$$
\mathrm{K}_{\mathrm{m}}\left(\gamma_{2} \mathrm{R} \sin (\mathrm{H})\right) \sim \sqrt{\pi / 2 \gamma_{2} R \sin (\mathrm{H})} \mathrm{e}^{-\gamma_{2} R \sin (\mathrm{H})}
$$

There obtains the far field pressure approximation

$\mathrm{p}_{2} \sim \frac{j \rho_{2}}{2 \pi \rho_{1}} \int_{-\infty}^{\infty} \frac{\mathrm{J}_{\mathrm{m}}\left(\mu_{\mathrm{mn}}\right)\left(1+\alpha \mathrm{M}_{2} / \mathrm{k}_{2}\right)}{\left(\alpha+\mathrm{K}_{\mathrm{mn}}\right) \mathrm{L}_{+}^{*}(\alpha) \mathrm{L}_{-}^{*}\left(-\mathrm{K}_{\mathrm{mn}}\right) \mathrm{K}_{\mathrm{m}}^{\prime}\left(\gamma_{2}\right) \gamma_{2}^{3 / 2}} \sqrt{\pi / 2 \mathrm{R} \sin \mathrm{H})} \times$ 
$e^{-j R h(\alpha)} d \alpha$

(40b)

where $h(\alpha)$ is the free field propagation factor

$$
h(\alpha)=\alpha \cos (H)-j \gamma_{2} \sin (H) .
$$

The inversion of (40) can now be carried out by the method of stationary phase [15]. The point of stationary phase corresponds to the root of the equation

$$
h^{\prime}(\alpha)=0
$$

straightforward algebra yields the requisite root $\alpha_{0}$

$$
\alpha_{0}=\frac{\mathrm{k}_{2}}{\left(1-\mathrm{M}_{2}^{2}\right)}\left[\mathrm{M}_{2}-\frac{\cos \mathrm{H})}{\sqrt{\left.1-\mathrm{M}_{2}^{2} \sin ^{2} \mathrm{H}\right)}}\right]
$$

There obtains then the solution to the radiation field

$$
\mathrm{p}_{2} \sim \mathrm{J}_{\mathrm{m}}\left(\mu_{\mathrm{mn}}\right) \mathrm{D}_{\mathrm{mn}}(\mathrm{H}) \frac{\mathrm{e}^{-\mathrm{j} \mathrm{R} h\left(\alpha_{0}\right)}}{\mathrm{R}}
$$

$\mathrm{D}_{\mathrm{mn}}$ (H) being the directivity factor defined by

$$
D_{m n}=\frac{j \rho_{2}\left(1+\alpha_{0} M_{2} / k_{2}\right)\left(\alpha_{0}+K_{m n}\right)^{-1} \gamma_{2}^{-3 / 2}}{2 \rho_{1} L_{-}^{*}\left(-K_{m n}\right)\left|h_{0}^{\prime \prime}\left(\alpha_{0}\right)\right|^{\frac{1}{2}} L_{+}^{*}\left(\alpha_{0}\right) K_{m}^{\prime}\left(\gamma_{2}\right)} \frac{e^{-j \pi / 4}}{\sqrt{\operatorname{sin~H}}}
$$

and where after some algebra

$$
h_{0}^{\prime \prime}\left(\alpha_{0}\right)=-\frac{\left(1-M_{2}^{2} \sin ^{2}(H)\right)^{3 / 2}}{k_{2} \sin ^{2}(H)}
$$

The $-\pi / 4$ factor arising in the directivity factor from the negative sign on $\mathrm{h}_{0}^{\prime \prime}\left(\alpha_{0}\right)$

and

$$
\gamma_{2}=\frac{-j k_{2} \sin (\mathrm{H})}{\sqrt{1-M_{2}^{2} \sin ^{2}(\mathrm{H})}}
$$

The kernel functions $L_{+}^{*}(\alpha)$ and $L_{-}^{*}(\alpha)$ being

$$
\begin{aligned}
& L_{+}^{*}(\alpha)=\left[\frac{\left(1+\alpha M_{1} / k_{1}\right)^{2}}{\left(1+\alpha M_{2} / k_{2}\right)}\right]\left[\frac{\tilde{k}_{2}+\tilde{\alpha}_{02}}{\tilde{k}_{2}-\tilde{\alpha}_{02}} \frac{\tilde{k}_{1}-\tilde{\alpha}_{01}}{\tilde{k}_{1}+\tilde{\alpha}_{01}}\right]^{m / 2} \times
\end{aligned}
$$

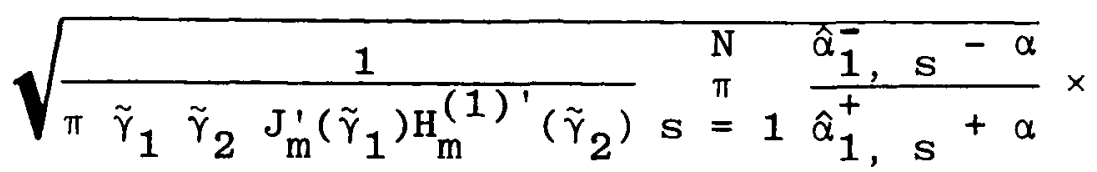




$$
\begin{aligned}
& \mathrm{T}_{+}^{*}(\alpha) \exp \left\{\frac{\tilde{\tilde{\alpha}}_{01}}{2}-\frac{\gamma_{2}}{2}-\frac{\mathrm{g}\left(\tilde{\alpha}_{02}\right)}{2}+\frac{\gamma_{1}}{\pi} \operatorname{arc} \cos \left(\tilde{\alpha}_{01} / \tilde{\mathrm{k}}_{1}\right)\right. \\
& \left.+j Y_{1}(\alpha)-\frac{j \pi}{4}\right\} \\
& \mathrm{L}_{-}^{*}(\alpha)=\left[\frac{\tilde{\mathrm{k}}_{2}-\tilde{\alpha}_{02}}{\tilde{\mathrm{k}}_{2}+\tilde{\alpha}_{02}} \frac{\tilde{\mathrm{k}}_{1}+\tilde{\alpha}_{01}}{\tilde{\mathrm{k}}_{1}-\tilde{\alpha}_{01}}\right]^{\mathrm{m} / 2} \times \\
& \sqrt{\frac{1}{\pi \tilde{\gamma}_{1} \tilde{\gamma}_{2} J_{m}^{\prime}\left(\tilde{\gamma}_{1}\right) H_{m}^{(1)^{\prime}\left(\tilde{\gamma}_{2}\right)} s} \stackrel{N}{=} \frac{\hat{\alpha}_{1, s}^{+}+\alpha}{\hat{\alpha}_{1, s}^{-}-\alpha}} \times \\
& \mathrm{T}_{-}(\alpha) \exp \left\{-\frac{\tilde{\alpha}_{01}}{2}-\frac{\gamma_{2}}{2}+\frac{\mathrm{g}\left(\tilde{\alpha}_{02}\right)}{2}+\frac{\gamma_{1}}{\pi}\left(\pi-\arccos \left(\tilde{\alpha}_{01} / \tilde{\mathrm{k}}_{1}\right)\right)\right. \\
& \left.-j Y_{1}(\alpha)-\frac{j \pi}{4}\right\}
\end{aligned}
$$

II.3.b Evaluation of the Conversion Coefficients of The conversion coefficients are deduced from the inversion

$p_{1}=\frac{j}{2 \pi} \int_{-\infty}^{\infty} \frac{\left(1+\alpha M_{1} / k_{1}\right)^{2}}{\left(1+\alpha M_{2} / k_{2}\right)} \frac{J_{m}\left(\mu_{m n}\right) L_{-}^{*-1}\left(-K_{m n}\right)}{\left(\alpha+K_{m n}\right) L_{+}^{*}(\alpha)} \frac{I_{m}\left(\gamma_{1} r\right)}{\gamma_{1} I_{m}^{\prime}\left(\gamma_{1}\right)} e^{-j \alpha x} d \alpha$

with the closure contour in the upper half plane. There are no branch points, only the simple zeros of $\gamma_{1} I_{m}^{\prime}\left(\gamma_{1}\right)$. Denote these zeros, obtained by solving the equation $\mathrm{m}_{\gamma_{1}}=j \mu_{\mathrm{ms}}$, by $\mathrm{K}_{\mathrm{ms}}$ to obtain for the pressure field within the duct in the limit $x \rightarrow-\infty$

$$
p_{1}=J_{m}\left(\mu_{m n}\right)\left[\frac{J_{m}\left(\mu_{m n} r\right)}{J_{m}\left(\mu_{m n}\right)} e^{j K_{m n} x}\right.
$$




$$
\left.+\sum_{s}^{\infty} \mathrm{R}_{\mathrm{ns}}^{\mathrm{mi}} \frac{\mathrm{J}_{\mathrm{m}}\left(\mu_{\mathrm{ms}} r\right)}{J_{m}\left(\mu_{\mathrm{ms}}\right)} e^{-j \mathrm{~K}_{\mathrm{ms}} \mathrm{x}}\right]
$$
where $\mathrm{R}_{\mathrm{ns}}^{\mathrm{m}}$ is the $\mathrm{m}^{\text {th }}$ mode conversion coefficient of the $\mathrm{n}^{\text {th }}$
radial mode into the $\mathrm{s}^{\mathrm{th}}$

$$
\mathrm{R}_{\mathrm{ns}}^{\mathrm{m}}=\frac{\left(1+\mathrm{K}_{\mathrm{ms}} \mathrm{M}_{1} / \mathrm{k}_{1}\right)^{2}}{\left(1+\mathrm{K}_{\mathrm{ms}} \mathrm{M}_{2} / \mathrm{k}_{2}\right)} \frac{\mathrm{-}^{-1}\left(-\mathrm{K}_{\mathrm{mn}}\right) \mathrm{L}_{+}^{*-1}\left(\mathrm{~K}_{\mathrm{ms}}\right)\left(1-\mathrm{m}^{2} / \mu_{\mathrm{ms}}^{2}\right)^{-1}}{\left(\mathrm{~K}_{\mathrm{ms}}+\overline{\mathrm{K}}_{\mathrm{mn}}\right)\left(\mathrm{k}_{1} \mathrm{M}_{1}-\mathrm{K}_{\mathrm{ms}}\left(1-\bar{M}_{1}^{2}\right)\right)}
$$

and

$$
\mathrm{K}_{\mathrm{ms}}=\frac{\mathrm{k}_{1} \mathrm{M}_{1}+\sqrt{\mathrm{k}_{1}^{2}-\mu_{\mathrm{ms}}^{2}\left(1-\mathrm{M}_{1}^{2}\right)}}{\left(1-\mathrm{M}_{1}^{2}\right)}
$$

$\mathrm{K}_{\mathrm{ms}}$ being the $\mathrm{s}^{\text {th }}$ reflected mode propagation constant, with the plus sign on the radical being picked for the upper half plane.

\section{II.4 Influence of a Doubly Infinite Centerbody}

Suppose now that the duct inner wall is a doubly infinite cylinder radius $\sigma$, then adopt for $p_{1}$ and $p_{2}$ the representations

$$
\begin{gathered}
\mathrm{p}_{1}=j \rho_{1} \omega \int_{-\infty}^{\infty} A_{+}(\alpha)\left(1+\alpha \mathrm{M}_{2} / \mathrm{k}_{2}\right) \times \\
\frac{\mathrm{I}_{\mathrm{m}}\left(\gamma_{1} r\right) \mathrm{K}_{\mathrm{m}}^{\prime}\left(\gamma_{1} \sigma\right)-\mathrm{K}_{\mathrm{m}}\left(\gamma_{1} r\right) I_{m}^{\prime}\left(\gamma_{1} \sigma\right)}{\gamma_{1}\left[I_{m}^{\prime}\left(\gamma_{1}\right) K_{m}^{\prime}\left(\gamma_{1} \sigma\right)-K_{m}^{\prime}\left(\gamma_{1}\right) I_{m}^{\prime}\left(\gamma_{1} \sigma\right)\right]} e^{-j \alpha x} d \alpha \\
p_{2}=j \rho_{2} \omega \int_{-\infty}^{\infty} B_{+}(\alpha) \frac{\left(1+\alpha M_{2} / k_{2}\right) K_{m}\left(\gamma_{2} r\right)}{\gamma_{2} K_{m}^{\prime}\left(\gamma_{2}\right)} e^{-j \alpha x} d \alpha
\end{gathered}
$$

Before proceeding further, let us introduce some shorthand notation to simplify otherwise bulky expressions involving cross-products of Bessel functions which we shall repeatedly encounter in the annular duct calculations. Specifically, we standardize on the notation of equations (9.1.32) of reference [14]. However, since several of the symbols have been used previously in a different context, we shall use asterisks to indicate the difference. In addition, to avoid ambiguity we will always specifically indicate the arguments involved. Hence note the following definitions:

$$
q_{m}^{*}(a, b)=J_{m}(a) Y_{m}^{\prime}(b)-J_{m}^{\prime}(b) Y_{m}(a)
$$




$$
\begin{aligned}
& r_{m}^{*}(a, b)=J_{m}^{i}(a) Y_{m}(b)-J_{m}(b) Y^{\prime}(a) \\
& s_{m}^{*}(a, b)=J_{m}^{\prime}(a) Y_{m}^{\prime}(b)-J_{m}^{\prime}(b) Y_{m}^{\prime}(a)
\end{aligned}
$$

Using the above shorthand notation, the incident wave can be expressed as

$$
p_{i n c}=q_{m}^{*}\left(\bar{\mu}_{m n} r, \bar{\mu}_{m n} \sigma\right) e^{j K_{m n}^{\sigma} x}
$$

Then repeating the earlier steps, the Wiener-Hopf kernel $L(\alpha)$ may be rewritten in the form

$$
\begin{aligned}
& L(\alpha)=[L(\alpha)]\left[\left\{\frac{\rho_{2}}{\rho_{1}} \frac{\gamma_{1}}{\gamma_{2}} \frac{K_{m}\left(\gamma_{2}\right) I_{m}^{\prime}\left(\gamma_{1}\right)}{K_{m}\left(\gamma_{2}\right) I_{m}\left(\gamma_{1}\right)} \frac{\left(1+\alpha M_{2} / k_{2}\right)^{2}}{\left(1+\alpha M_{1} / k_{1}\right)^{2}}-\right.\right. \\
& \left(\frac{1-\frac{K_{m}\left(\gamma_{1}\right)}{K_{m}^{\prime}\left(\gamma_{1} \sigma\right)} \frac{I_{m}^{\prime}\left(\gamma_{1} \sigma\right)}{I_{m}\left(\gamma_{1}\right)}}{1-\frac{K_{m}^{\prime}\left(\gamma_{1}\right)}{K_{m}^{\prime}\left(\gamma_{1} \sigma\right)} \frac{I_{m}^{\prime}\left(\gamma_{1} \sigma\right)}{I_{m}^{\prime}\left(\gamma_{1}\right)}}\right) \int\left\{\frac{\rho_{2}}{\rho_{1}} \frac{\gamma_{1}}{\gamma_{2}} \frac{K_{m}\left(\gamma_{2}\right) I_{m}^{\prime}\left(\gamma_{1}\right)}{K_{m}^{\prime}\left(\gamma_{2}\right) I_{m}\left(\gamma_{1}\right)} \times\right. \\
& \left.\frac{\left(1+\alpha \mathrm{M}_{2} / \mathrm{k}_{2}\right)^{2}}{\left(1+\alpha \mathrm{M}_{1} / \mathrm{k}_{1}\right)^{2}}-1\right\}
\end{aligned}
$$

The first square bracketed term is precisely the kernel $L(\alpha)$ decomposed for the cylindrical duct to which reference may be made. There remains the last factor which we designate as $W(\alpha)$ for convenience. Again adopt the Koiter approximation $W^{*}(\alpha)$

$$
W *(\alpha) \sim \frac{\bar{\delta} \bar{B}}{\bar{\lambda}} \frac{\alpha^{2}+\alpha+\bar{\delta} / \bar{B}}{\alpha^{2}+\alpha+\bar{\delta} / \bar{\lambda}}
$$

where by matching the function in the limits $\alpha \rightarrow 0$ and $\alpha \rightarrow \infty$ and the first derivative as $\alpha \rightarrow 0$ we obtain

$$
\frac{\bar{\delta} \bar{B}}{\bar{\lambda}}=\lim _{\alpha \rightarrow \infty} W(\alpha)=1
$$

and

$$
\begin{aligned}
& \bar{\delta}=W(0) \\
& \bar{\delta} / \bar{\lambda}=\frac{W(\infty)-W(0)}{W^{\prime}(0)}
\end{aligned}
$$




$$
\bar{\delta} / \bar{B}=\bar{\delta} / \bar{\lambda} \frac{W(0)}{W(\infty)}
$$

Now repeating the earlier calculations for the radiation field and conversion coefficients obtain the result, in the far field for $\mathrm{p}_{2}\left(\alpha_{0}\right.$ being defined by (41))

$$
\mathrm{p}_{2} \sim \mathrm{q}_{\mathrm{m}}^{*}\left(\bar{\mu}_{\mathrm{mn}}, \bar{\mu}_{\mathrm{mn}} \sigma\right) \mathrm{D}_{\mathrm{mn}}^{\sigma}(\text { (日) }) \frac{\mathrm{e}^{-j R h\left(\alpha_{0}\right)}}{\mathrm{R}}
$$

with the directivity factor $\mathrm{D}_{\mathrm{mn}}^{\sigma}(\mathrm{G})$

$$
\mathrm{D}_{\mathrm{mn}}^{\sigma}(\text { (1) })=\frac{j \rho_{2}}{2 \rho_{1}} \frac{\left(1+\alpha_{0} \mathrm{M}_{2} / \mathrm{k}_{2}\right)\left(\alpha_{0}+\mathrm{K}_{\mathrm{mn}}^{\sigma}\right)^{-1} \gamma_{2}^{-3 / 2} \mathrm{e}^{-j \pi / 4}}{L_{-}^{*}\left(-K_{\mathrm{mn}}^{\sigma}\right)\left|h^{\prime \prime}\left(\alpha_{0}\right)\right|^{\frac{1}{2}} L_{+}^{*}\left(\alpha_{0}\right) K_{m}^{\prime}\left(\gamma_{2}\right) \sqrt{\sin }(\mathbb{1})}
$$

where $\bar{\mu}_{\mathrm{mn}}$ now are the zeros of

$$
\bar{\mu}_{\mathrm{m}}^{2} \mathrm{~s}_{\mathrm{m}}^{*}\left(\sigma, \bar{\mu}_{\mathrm{m}} \sigma\right)=0
$$

$\bar{\mu}_{p, ~}=0 \mathrm{~m} \neq 0$ are trivial and are not included in the set
of admissible eigenvalues.

To determine the pressure field within the duct, invert (49) with a contour closed in the upper half $\alpha$ plane. Again there are no branch points and the inversion is carried out by summing the residues at the zeros of $\gamma_{1}\left[I_{m}^{\prime}\left(\gamma_{1}\right) K_{m}^{\prime}\left(\sigma \gamma_{1}\right)-\right.$ $\left.K_{m}^{\prime}\left(\gamma_{1}\right) I_{m}^{\prime}\left(\sigma \gamma_{1}\right)\right]$. Denote the values of $\alpha$ at these points by $K_{m}^{\sigma}$ Then using the cross-product notation of [14], as defined above, ${ }^{\circ}$ the pressure field within the duct is given, away from the exit plane of the duct, by

$$
\begin{aligned}
\mathrm{p}_{1}= & \mathrm{q}_{\mathrm{m}}^{*}\left(\bar{\mu}_{\mathrm{mn}}, \bar{\mu}_{\mathrm{mn}} \sigma\right)\left[\frac{\mathrm{q}_{\mathrm{m}}^{*}\left(\bar{\mu}_{\mathrm{mn}} \mathrm{r}, \bar{\mu}_{\mathrm{mn}} \sigma\right)}{\mathrm{q}_{\mathrm{m}}^{*}\left(\bar{\mu}_{\mathrm{mn}}, \bar{\mu}_{\mathrm{mn}} \sigma\right)} \mathrm{e}^{\mathrm{j} \mathrm{K}_{\mathrm{mn}}^{\sigma} \mathrm{x}}\right. \\
& +\sum_{\mathrm{s}} \stackrel{\sum_{=}^{\infty}}{1} \mathrm{R}_{\mathrm{ns}}^{\mathrm{m}}\left\{\frac{\mathrm{q}_{\mathrm{m}}^{*}\left(\bar{\mu}_{\mathrm{ms}} \mathrm{r}, \bar{\mu}_{\mathrm{ms}} \sigma\right)}{\left.\left.\mathrm{q}_{\mathrm{m}}^{*\left(\bar{p}_{\mathrm{ms}}, \bar{p}_{\mathrm{ms}} \sigma\right)}\right\} \mathrm{e}^{-j \mathrm{~K}_{\mathrm{ms}}^{\sigma} \mathrm{x}}\right]}\right.
\end{aligned}
$$

where the conversion coefficients $R_{n s}^{m}$ are now defined as

$$
\begin{aligned}
\mathrm{R}_{\mathrm{ns}}^{\mathrm{m}} & =\frac{\left(1+\mathrm{K}_{\mathrm{ms}}^{\sigma} \mathrm{M}_{1} / \mathrm{k}_{1}\right)}{\left(1+\mathrm{K}_{\mathrm{ms}}^{\sigma} \mathrm{M}_{2} / \mathrm{k}_{2}\right)} \frac{\mathrm{L}_{-}^{*-1}\left(-\mathrm{K}_{\mathrm{mn}}^{\sigma}\right) \mathrm{L}_{+}^{*-1}\left(\mathrm{~K}_{\mathrm{ms}}^{\sigma}\right)\left(1-\mathrm{m}^{2} / \bar{\mu}_{\mathrm{ms}}^{2}\right)^{-1}}{\left(\mathrm{~K}_{\mathrm{ms}}^{\sigma}+\mathrm{K}_{\mathrm{mn}}^{\sigma}\right)\left(\mathrm{k}_{1} \mathrm{M}_{1}-\mathrm{K}_{\mathrm{ms}}^{\sigma}\left(1-\mathrm{M}_{1}^{2}\right)\right)} \times \\
& \left\{\frac{1}{1+\sigma \frac{\left(\bar{\mu}_{\mathrm{ms}}^{2}-\mathrm{m}^{2} / \sigma^{2}\right)}{\left(\bar{\mu}_{\mathrm{ms}}^{2}-\mathrm{m}^{2}\right)} \frac{\mathrm{r}_{\mathrm{m}}^{*}\left(\bar{\mu}_{\mathrm{ms}}, \bar{\mu}_{\mathrm{ms}} \sigma\right)}{\mathrm{q}_{\mathrm{m}}^{*}\left(\bar{\mu}_{\mathrm{ms}}, \bar{\mu}_{\mathrm{ms}} \sigma\right)}}\right\}
\end{aligned}
$$




\section{II.5 The InIet Probiem}

Strictly speaking, the plug flow jet approximation is totally incorrect in the inlet mode. However allowing the license, one can treat such a formulation as a crude first approximation, especially in view of the intractability of the problem with a sink flow. For the inlet mode, the flow duct is considered to be located at $r=1$ and $x>0$. Again, both $M_{1}$ and $M_{2}$ are taken to be positive.

The inlet problem now is very similar to the exhaust problem in that the governing equation and boundary conditions are quite similar to those already considered. Hence only an outline of the solution is given below. Recalling that

$$
-\frac{1}{\rho} \frac{\partial p}{\partial r}=\left[\frac{\partial}{\partial t}+M c \frac{\partial}{\partial x}\right]^{2} \eta
$$

Let

$$
\begin{gathered}
\eta_{1}=\int_{-\infty}^{\infty} \frac{A_{-}(\alpha) I_{m}^{\prime}\left(\gamma_{1} r\right) e^{-j \alpha x} d \alpha}{I_{m}^{\prime}\left(\gamma_{1}\right)} \\
p_{1}=\rho_{1} \omega^{2} \int_{-\infty}^{\infty} \frac{A_{-}(\alpha)\left(1+\frac{\alpha M_{1}}{k_{1}}\right)^{2} e^{-j \alpha x} I_{m}\left(\gamma_{1} r\right) d \alpha}{\gamma_{1} I_{m}^{\prime}\left(\gamma_{1}\right)}
\end{gathered}
$$

(It is readily verified that (60) and (61) satisfy (59).)

$$
n_{\text {inc }}=\frac{\exp \left(-j K_{m n}^{*} x\right) \mu_{m n} J_{m}^{\prime}\left(\mu_{m n} r\right)}{\rho_{1} \omega^{2}\left\{1+\frac{M_{1} K_{m n}^{*}}{k_{1}}\right\}^{2}}
$$

where

$$
\mathrm{K}_{\mathrm{mn}}^{*}=\frac{\sqrt{\mathrm{k}_{1}^{2}-\left(1-\mathrm{M}_{1}^{2}\right) \mu_{\mathrm{mn}}^{2}}+\mathrm{k}_{1} \mathrm{M}_{1}}{\left(1-\mathrm{M}_{1}^{2}\right)}
$$

and

$$
\mathrm{p}_{\text {inc }}=\exp \left(-j \mathrm{~K}_{\mathrm{mn}}^{*} \mathrm{x}\right) \mathrm{J}_{\mathrm{m}}\left(\mu_{\mathrm{mn}} \mathrm{r}\right)
$$

In region II:

$$
n_{2}=\int_{-\infty}^{\infty} \frac{B_{-}(\alpha) K_{m}^{\prime}\left(\gamma_{2} r\right) e^{-j \alpha x} d \alpha}{K_{m}^{\prime}\left(\gamma_{2}\right)}
$$




$$
p_{2}=\rho_{2} \omega^{2} \int_{-\infty}^{\infty} \frac{B_{-}(\alpha)\left[1+\frac{M_{2} \alpha}{k_{2}}\right]^{2} e^{-j \alpha \bar{x}} K_{m}\left(\gamma_{2} r\right) d \alpha}{\gamma_{2} K_{m}^{\prime}\left(\gamma_{2}\right)}
$$

As before (62) indicates that $n_{\text {inc }}=0$ for $r=1$ for all $x$. Hence by (65), (60), the Wiener-Hopf technique and Liouvilie's theorem:

$$
A_{-}(\alpha)=B_{-}(\alpha)
$$

Application of the pressure boundary condition for $x<0$ yields:

$$
\begin{gathered}
{\left[\frac{\rho_{2}\left(1+\frac{\alpha M_{2}}{k_{2}^{2}}\right)^{2} K_{m}\left(\gamma_{2}\right)}{\rho_{1} \gamma_{2} K_{m}^{\prime}\left(\gamma_{2}\right)}-\frac{\left(1+\frac{\alpha M_{1}}{k_{1}}\right)^{2} I_{m}\left(\gamma_{1}\right)}{\gamma_{1} I_{m}^{\prime}\left(\gamma_{1}\right)}\right] A_{-}(\alpha)} \\
+\frac{j J_{m}\left(\mu_{m n}\right)}{2 \pi\left(\alpha-K_{m n}^{*}\right) \rho_{1} \omega^{2}}=H_{+}(\alpha)
\end{gathered}
$$

Again if we let

$$
\begin{aligned}
\bar{L}(\alpha) & =\left[\frac{\rho_{2}\left(1+\frac{\alpha M_{2}}{k_{2}^{2}}\right)^{2} K_{m}\left(\gamma_{2}\right)}{\rho_{1} \gamma_{2} K_{m}^{\prime}\left(\gamma_{2}\right)}\right. \\
& \left.-\frac{\left(1+\frac{\alpha M_{1}}{k_{1}^{2}} I_{m}\left(\gamma_{1}\right)\right.}{\gamma_{1} I_{m}^{\prime}\left(\gamma_{1}\right)}\right]
\end{aligned}
$$

and if $\overline{\mathrm{L}}(\alpha)$ can be factored as $\overline{\mathrm{L}}_{+}(\alpha) \overline{\mathrm{L}}_{-}(\alpha)$, then the solution of (68) is that

$$
A_{-}(\alpha)=\frac{-j J_{m}\left(\mu_{m n}\right)}{\rho_{1} \omega^{2} 2 \pi\left(\alpha-K_{m n}^{*}\right) \bar{L}_{+}\left(K_{m n}^{*}\right) \bar{L}_{-}(\alpha)}
$$

(70) completes the formal solution to the inlet problem.

Note from (69) and (19) that

$$
\overline{\mathrm{L}}(\alpha) /\left(1+\frac{\alpha \mathrm{M}_{2}}{\mathrm{k}_{2}}\right)=\mathrm{L}(\alpha)
$$

Since $\left(1+\alpha M_{2} / k_{2}\right)$ is clearly a plus function we have immediately that 


$$
\left.\begin{array}{c}
\overline{\mathrm{L}}_{-}(\alpha)=\mathrm{L}_{-}(\alpha) \\
\overline{\mathrm{L}}_{+}(\alpha)=\left(1+\alpha \mathrm{M}_{2} / \mathrm{k}_{2}\right) \mathrm{L}_{+}(\alpha)
\end{array}\right\}
$$

and

Equation (71) shows that any formula or method to factorize $\mathrm{L}(\alpha)$ will immediately yield the factorization of $\overline{\mathrm{L}}(\alpha)$.

We wish to note that for the inlet problem if there is a uniform flow over the entire plane the application of the boundary condition that $\Delta p(x)=0$ for $x<0, r=1$ (where $\Delta p$ denotes the jump in acoustic pressure across the phantom duct extension described by $r=1$ and $x<0$ ) automatically ensures that $\Delta \phi$ is also zero across such a surface. This is seen by noting that if we write

$$
\Delta p(x)=\int_{-\infty}^{\infty} P(\alpha) e^{-j \alpha x} d \alpha
$$

then $\mathrm{p}=0$ for $\mathrm{x}<0$ implies that $\mathrm{P}(\alpha)=\mathrm{P}_{+}(\alpha)$, i.e. that $P(\alpha)$ is analytic in an upper half plane. But the relation between $\Phi(\alpha)$, the Fourier transform of $\Delta \phi(x)$, and $P_{+}(\alpha)$ is (for the case of uniform flow)

$$
\Phi(\alpha)=\frac{-j \mathrm{P}_{+}(\alpha)}{\rho \omega\left[1+\frac{\alpha \mathrm{M}}{\mathrm{k}}\right]}
$$

Since the denominator in the expression for $\Phi(\alpha)$ has only a lower half plane zero (at $\alpha=-k / M$ ), it is clear that $\Phi(\alpha)$ is also analytic in an upper half plane (i.e. $\Phi(\alpha)=\Phi_{+}(\alpha)$ ). This means that $\Delta \phi$ will be zero for $x<0$ and $r=1$. We have verified that our solutions to the inlet and exhaust problems are compatible with other solutions for the case of uniform flow.

\section{DISCUSSION OF SAMPLE CALCULATIONS}

At the outset, let us clearly designate the convention we will be using in labeling the various modes. We shall always designate the lowest zero of a given lobe order $m$ as 1 , i.e. $\mathrm{n}=1$ will be the lowest propagated mode of lobe number $\mathrm{m}$. Then, a label such as, the $(\mathrm{m}, \mathrm{n})$ mode will mean an $\mathrm{m}$ lobed spinning wave and the $n^{\text {th }}$ radial order. ( $\left.0, n\right)$ waves will be the symmetric waves, and the plane wave will be $(0,1)$. When we designate the phase of the reflected wave $\theta_{\text {ns }}$, this will be taken to mean the $s^{\text {th }}$ reflected radial mode of an $(m, n)$ incident wave.

In the development of the theoretical model, a number of approximations were made, some physical and some mathematical. Each of these will clearly have their impact. Consider first the physical approximations: The most crucial physical approximation is the exclusion of any physical inhomogeneities 
such as turbulence and neglect of jet spread and mixing. While this rendered the mathematics tractable, the use of the uniform plug flow model entailed the use of the particle displacement match boundary condition. Similarly, the thickness of the exhaust duct and the termination of the annular centerbody were excluded. The primary effect of these approximations is to render the phase calculations inaccurate, since the phase is particularly sensitive to such termination effects. The least effect of such approximations is on the amplitude of the radiation pattern. Thus it would appear that in phase synthesis of a radiation pattern composed of a set of flow duct modes is practically difficult if not rendered meaningless. On the other hand the restrictive effects of the mathematical approximations introduced by the Carrier-Kojter procedure are probably much less (except near the axis as (H) $\rightarrow 0$ for the radiation pattern, as discussed below) provided one carefully observes the rules devised by Koiter [9] and Carrier [10]. Indeed in our calculations this approximation is restricted only to the equivalent of the Wronskian normalized by the uniform flow Wronskian, so that for uniform flow and no flow, our theory is exact. In what follows, we shall try to illustrate these points by concrete comparisons, especially those involving experimental measurements.

There are several interesting features displayed by the solutions such as equations $(42 a, b)$. The most obvious is the convective amplification due to the main jet, $\left(1+\alpha \mathrm{M}_{1} / \mathrm{k}_{1}\right)^{2}$, and the weakening of that factor by the external flow, $\left(1+\alpha M_{2} / k_{2}\right)^{2}$. The second feature relates to the zone of relative silence defined by

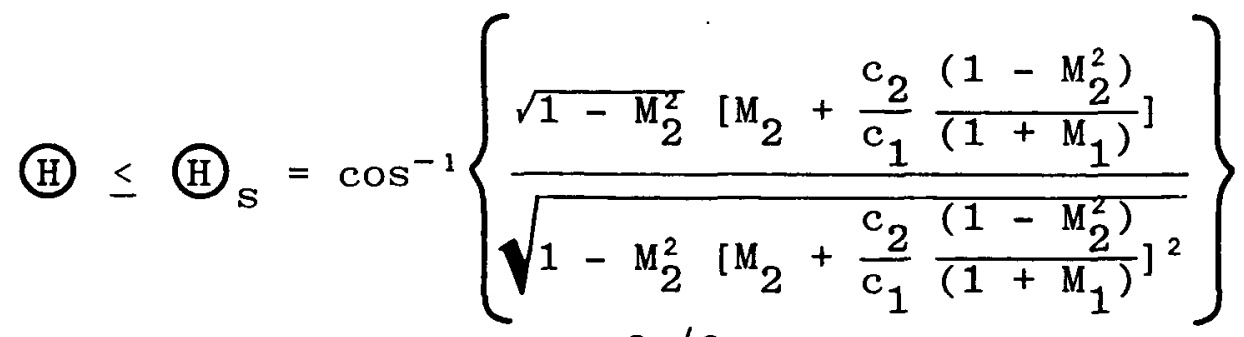

$$
\begin{aligned}
& \text { or for } \left.M_{2}=0 \quad \text { (H) }\right)_{s}=\cos ^{-1}\left\{\frac{c_{2} / c_{1}}{1+M_{1}}\right\}
\end{aligned}
$$

within the zone of relative silence the signal suffers attenuation due to the exponential factor $\exp \left\{\gamma_{1} / \pi \operatorname{arc} \cos \left(\tilde{\alpha}_{01} / \tilde{\mathrm{k}}_{1}\right)\right\}$, which results in a cusp at (H) $=$ (H) for the plane wave and nodes for the higher modes. The cusp has been previously observed by Gott1ieb [16].

In the case of uniform flow $\left(M_{1}=M_{2}\right)$, the limit of the solution for $p_{2}$ as (H) $\rightarrow 0$ is the same as the solution obtained for $p_{1}$ (taking the case of the exhaust flow for concreteness) with the closure contour in the lower half $\alpha$ plane. Such is not the case for $M_{1} \neq M_{2}$ in the present solution. Indeed, the pressure distribution within the jet, as represented by, for example, (46) displays a zero pressure on the axis which is not reproduced from the far field radiation pattern as $(H) \rightarrow$ 0 . This appears to be the consequence of the Carrier-Koiter approximation. 
Consider now the solutions for the symmetric modes, $(0$, $n)$. For the case of no flow and plane wave incident, our solution is exact as is illustrated by Figures 7, 8, 9. These results may be compared with the results of Levine and Schwinger [2] among others.

More useful comparisons, however, are offered by the experimental data of Plumblee et al. [6] and Mechel et al. [7]. Figures 10,11 compare the flow and no flow data and includes the calculated effect of inclusion or exclusion of a hub. Plumblee's data correspond to a monochromatic exhaust mode and a hub which is terminated in the duct exhaust plane, so that the calculated situation does not correspond exactly. The measurements have been made with great care. Not surprisingly, the no flow results show a reasonable agreement, however the cusped lobe of the calculation with flow is smoothed into an outward leaning cleaner lobe. Such a smoothing of the radiation pattern is to be expected in view of the scattering the radiated sound pattern by the intense turbulence of the spreading exhaust jet. This process is well illustrated by the calculations of Mani [17], thus note in particular Figures 5, 6, 7 of that reference. Nevertheless, the influence of the exhaust jet refraction is well brought out by both the data and calculations. Indeed accounting for the diffusive smoothing of the pattern, the peak direction does seem to be approximated. On the other hand, the influence of the hub is seen to be negligible.

Plumblee et al. [6] have also presented some measured radiation impedances. A sample comparison of these cases calculated on the basis of our theory are presented in Table I. The agreement, even in the case of no flow is somewhat less than satisfactory. This well illustrates the influence of the truncated hub in altering phase relations. Calculations without the hub included show no better agreement. What limited agreement there was with no flow, breaks down in the presence of flow. Thus the physical flow model appears to greatly exaggerate the acoustic mismatch between the duct and the surrounding, and the error, as will be seen presently, does not wholly originate of the Koiter approximation.

Before considering the data of Mechel et al. [7], recall that the use of the uniform plug flow model resulted in the use of the Miles [12] - Ribner [13] particle displacement match boundary condition. In essence, this boundary condition results from the assumption of a clearly defined flow interfacial streamline with the acoustic motion being transmitted from one region into another by the transverse perturbation of that clearly defined interfacial streamline. However, in the case of the ill-defined, highly turbulent flow of the jet boundary, the mean velocity changes "almost" smoothly across the interfacial region. In this case, noting the calculations of Mani [17], there appears to be little reason to presume the picture based on the Miles-Ribner argument. Interest in this question was recently revived by Ingard and Singhal [18] who presented intriguing experimental evidence on the coupling of a sound source with a turbulent flow duct. Their evidence, 
moreover seemed to favor the discarded notion of continuity of particle velocity rather than the displacement.

It was hoped for that the present calculations be useful in this regard concerning the exhaust jet. The thought was that the duct reflection amplitude is a function of the boundary condition used. Then a comparison of the low frequency prediction using the two boundary conditions when compared with low frequency flow duct experiments of a similar nature might sort out this thorny question. Indeed the data of Mechel et a1. [7] offer this opportunity, and it was a simple matter to modify our theory to include a particle velocity match at the jet interface.

Such a test, as discussed above, is offered in Figure 12. Both the boundary conditions show a similar trend and predict reflection coefficients higher than the uniform flow case. Neither calculations reproduce the peak in measured reflection coefficients, but both do reproduce the reflection coefficients to within an acceptable error. However the marginal variation in the calculation does not allow a critical judgment of the boundary condition. As a closing remark for this aspect of the discussion, it should be noted that the no flow results of Mechel et al. did reproduce the calculated results to a high degree of accuracy.

Finally, before proceeding to the discussion of asymmetric modes, the influence of the Mach number and flow mismatch are illustrated in Figure 13.

Consider now the asymmetric modes. In light of the above discussion, we shall concentrate on results for the radiation patterns. In Figure 14, a comparison between our theory and the experimental results of the phased array model of Plumblee et al. [6] is offered. A disconcerting feature of these data is the non-zero radiation amplitude on the axis even without flow. This suggests spurious effects not accounted for in the physical model on which the experiments are based. Similarly, the theoretically predicted lobe in the shadow zone of the jet cannot be picked up over the flow noise of the acoustic pickup.

A somewhat better agreement is apparent for the calculated $(4,1)$ mode in Figure 15. Plumblee's data have not been plotted on this figure and reference must be made to Figure 20 of that paper. However the refractive shifting of the pattern and the narrowing of the dominant lobe are both reproduced. Figure 15 also presents the effect of a weak external flow which may be interpreted also as a flight effect. The ultimate situation of the flight speed matching the jet speed being the uniform flow case. The effect of the weak external flow is to shift the pattern towards the axis and reduce the shadow zone lobe.

More dramatic example of shown in Figure 16. One sees axial convection at high Mach the jet Mach number effect is the dominant component of the radiation in the shadow zone and 
the sharp refraction towards the sideline. The flow enhanced frequency $\widetilde{\mathrm{k}}_{1}$ also increases the number of nodes. Compare this figure with the plane wave calculation in Figure 2 of Mani [5].

The physical model we have chosen is expected to better represent the exhaust conditions. However, the effect of flow inhomogeneities has been to weaken the correspondence. On the other hand in the inlet mode, the smoother sink flow is not represented by our model. Yet paradoxically, note the comparison of our calculations with the Crigler and Copeland [8] data. It would appear that the $53-62$ rotor/IGV interaction has resulted in a dominant $(9,2)$ mode. Compare too the phased array data of Pasko [19] with our calculations in Figure 18. In both the cases, we used a small external flow to weaken the degree of the flow mismatch.

In the turbine exhaust noise problem, the dominant flow mismatch is the temperature. From simple kinematic considerations, it is possible to illustrate the influence of the hot jet is to sharply beam the sound into the sideline. Thus Candel [20] has shown that a plane wave incident on a flow/ temperature interface at an angle $\theta_{1}$ is sharply refracted to an angle $\theta_{2}$

$$
\cos \theta_{2}=\frac{\left(c_{2} / c_{1}\right) \cos \theta_{1}}{1-M_{1} \cos \theta_{1}}
$$

with $\mathrm{M}_{2}=0$. Hence consider the radiation patterns of Figures 19,20 . Figure 20 is somewhat more meaningful since it corresponds to a physical case of fixed axial velocity and frequency of the upstream sound source.

\section{CONCLUSIONS}

In this study we have offered a method of calculation for spinning acoustic duct wave guide mode radiating through a flow mismatch. The convected wave equation was solved in part exactly, partly accounting for the asymmetry introduced by the flow mismatch into the mathematics by the Carrier-Koiter procedure. An attempt was made to compare the predictions of the current theory with realistic experimental models. It is concluded that the value of the calculations is primarily in explaining qualitative features of spinning mode radiation patterns. Real flow features not accounted for especially preclude the reproduction of phase information. Paradoxically therc appears to be a better qualitative agreement for the inlet mode for which the physical model is not really meant than the exhaust mode which we tried to approximate. This appears to be the result of relatively cleaner inflow conditions. An attempt was also made to test the issue of the flowacoustic boundary condition, but the result was not decisive. 


\section{REFERENCES}

1. Tyler, J. M. and Sofrin, T. G.: Axial Flow Compressor Noise Studies, SAE Trans., Vol. 70, 1962, pp. 309332 .

2. Levine, H. and Schwinger, J.: On the Radiation of Sound from an Unflanged Circular Pipe. Phys. Rev., Vol. 73, No. 4, Second Ser., Feb. 1948, pp. 383-406.

3. Carrier, G. F.: Sound Transmission from a Tube with Flow. Quart. Appl. Math., Vol. XIII, No. 4, Jan. 1956, pp. 457-561.

4. Lansing, D. L., Drischler, J. A. and Pusey, C. G.: Radiation of Sound from an Unflanged Circular Duct with Flow. Presented at 79 th Meeting of Acoustical Soc. Am., Atlantic City, 1970.

5. Mani, R.: Refraction of Acoustic Duct Waveguide Modes by Exhaust Jets. Quart. App1. Math., Vol. XXX, No. 4, Jan. 1973, pp. 501-520.

6. Plumblee, H. E. and Dean, P. D.: Sound Measurements Within and in the Radiated Field of an Annular Duct with Flow. J. of Sound Vib., Vol. 28, No. 4, 1973, pp. 715-735. (See also Lockheed-Georgia Company Contractor Report under NASA Contract NAS1-10472).

7. Mechel, von F., Schilz, W. und Dieta, J.: Akustische Impedanz Einer Luftdurchstromten Offnung, Acustica, Vol. 15, 1965, pp. 199-206.

8. Crigler, J. L. and Copeland, W. L.: Noise Studies of Inlet-Guide-Vane-Rotor Interaction of a Single Stage Axia1-Flow Compressor, NASA TN D-2962, Sept. 1965.

9. Koiter, W. T.: Approximate Solution of Wiener-Hopf Type Integral Equations with Applications, Koninkl. Ned. Akademie Wetenschappen, Amsterdam, Proc. B, Vol. 57, No. 5,1954 .

10. Carrier, G. F.: Useful Approximations in Wiener-Hopf Problems, J. Appl. Physics, Vol. 30, No. 11, 1959.

11. Noble, B.: Methods Based on the Wiener-Hopf Technique for the Solution of Partial Differential Equations, Pergammon Press, 1958.

12. Miles, J. W.: On the Reflection of Sound at an Interface of Relative Motion, J. Acoust. Soc. Am., 1957, Vol. 29 , pp. 226-228.

13. Ribner, H. S.: Reflection, Transmission and Amplification of Sound by a Moving Medium, J. Acoustic. Soc. Am., 1957, Vol. 29, pp. 435-441. 
14. Abramowitz, M. and Stegun, I. E.: Handbook of Mathematical Functions, NBS Applied Math Series No. 55, Nov. 1970.

15. Carrier, G. F., Krook, M. and Pearson, C. E.: Functions of a Complex Variable, McGraw-Hill Book Company, 1966 , pp. 272-275.

16. Gottlieb, P.: Sound Source Near a Velocity Discontinuity, J. Acoust. Soc. Am., 1960, Vol. 32, No. 9, pp. 11171122 .

17. Mani, R.: Diffusion of Radiation Patterns Due to Scattering By Random Inhomogeneities, J. Sound Vib., 1971, Vol. 17, No. 1, pp. 95-104.

18. Ingard, K. U. and Singha1, V. K.: Upstream and Downstream Sound Radiation in a Moving Fluid, MIT Quarterly Progress Report No. 108, 1973.

19. Pasko, W.: The Propagation of Discrete Frequency Noise From an Annular Duct with Non Uniform Flow, University of Mass. Ph. D. Thesis, May 1973.

20. Candel, S. M.: Acoustic Transmission and Reflections By a Shear Discontinuity Separating Hot and Cold Regions, J. Sound Vib., 1972, Vol. 24, No. 1, pp. 87-91. 


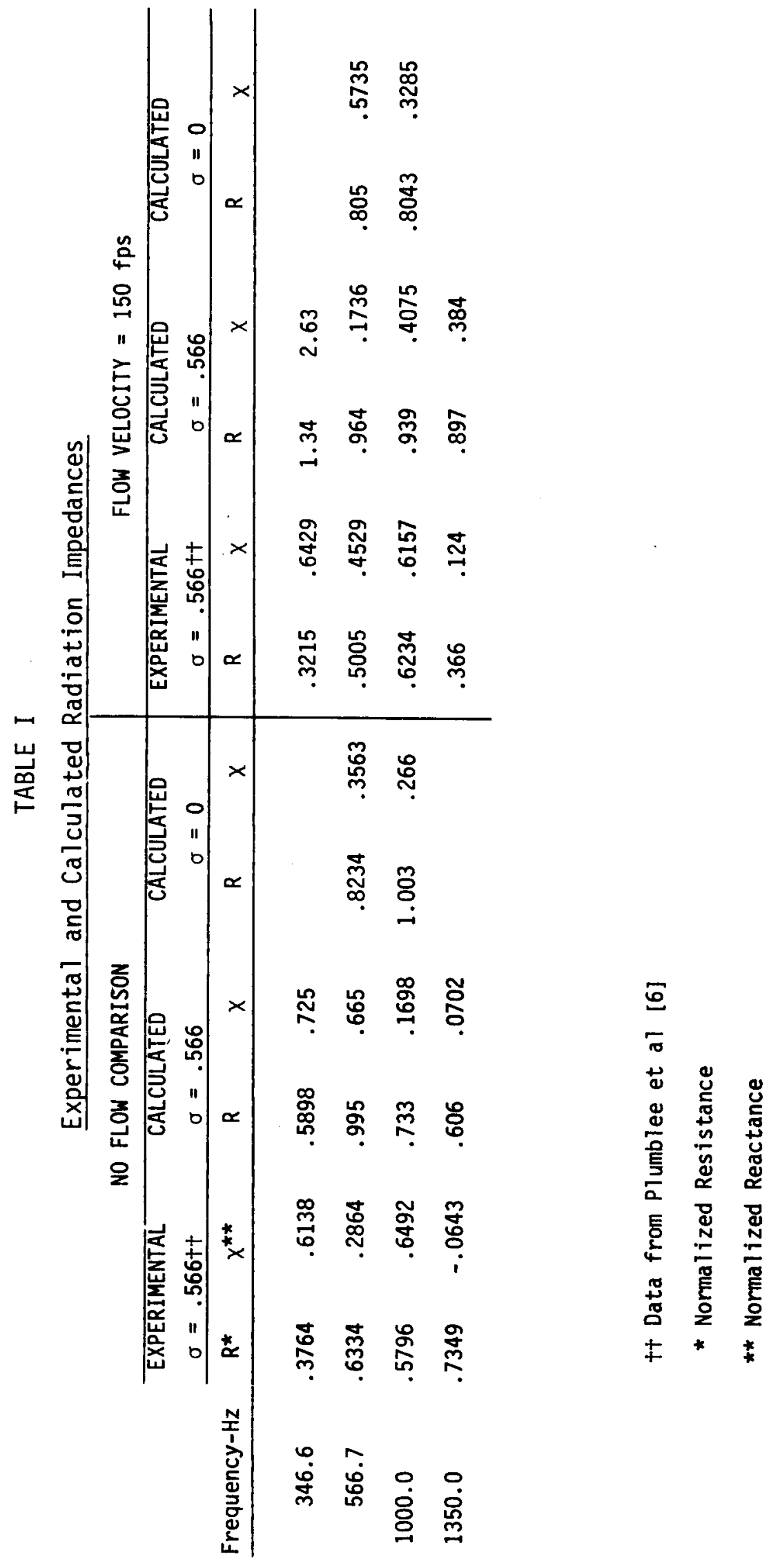




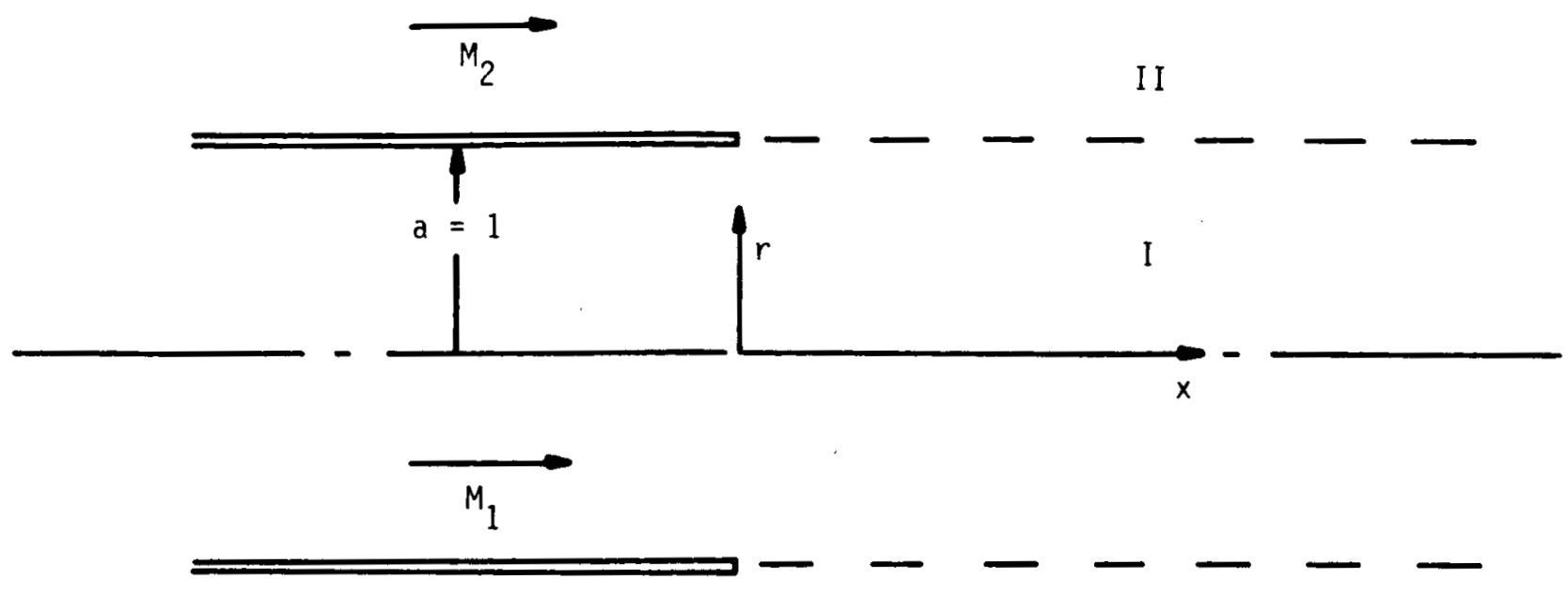

Figure 1 Schematic of the model problem. 


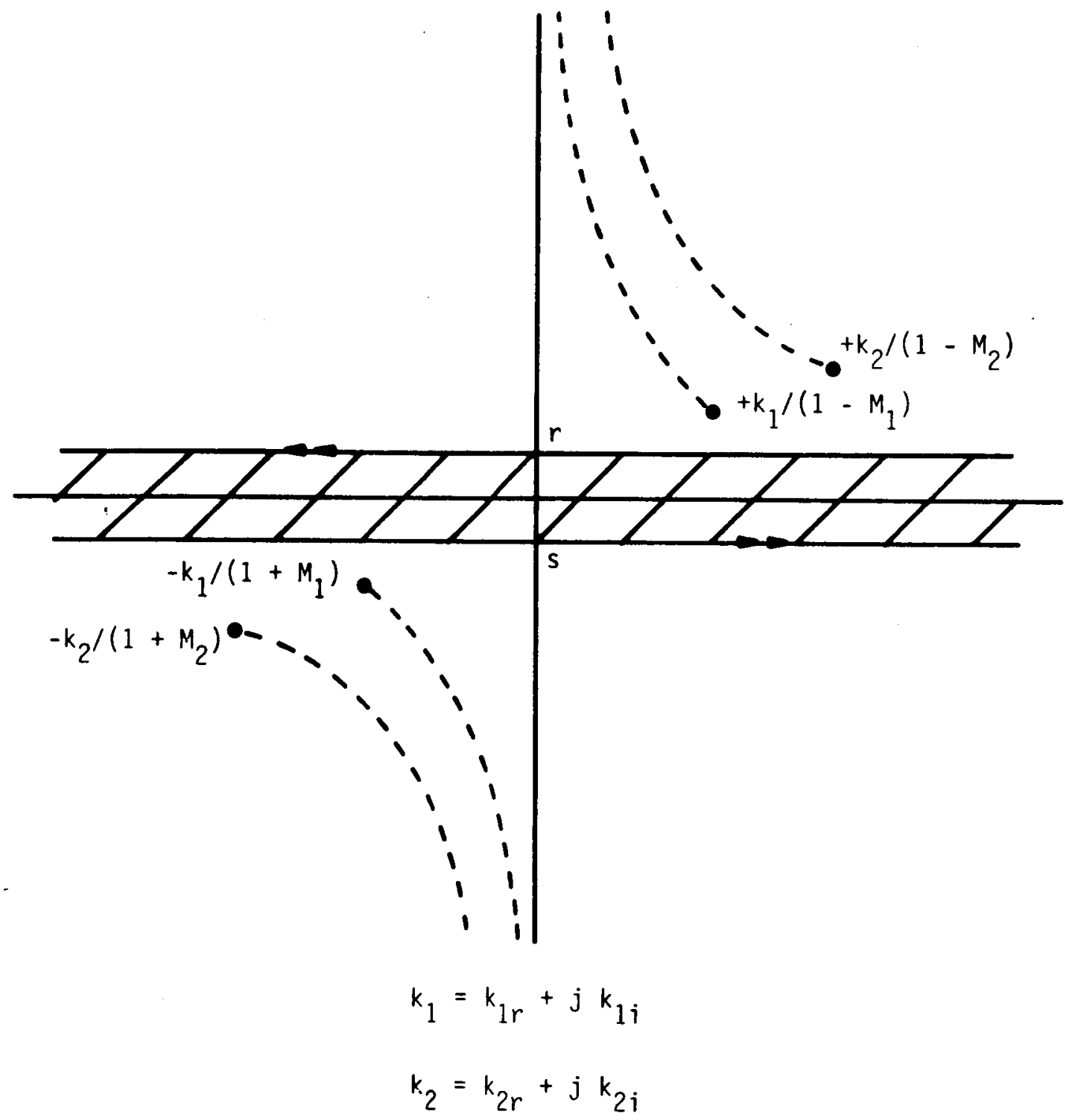

Figure 2 Branch cuts on the complex $\alpha$-plane. 


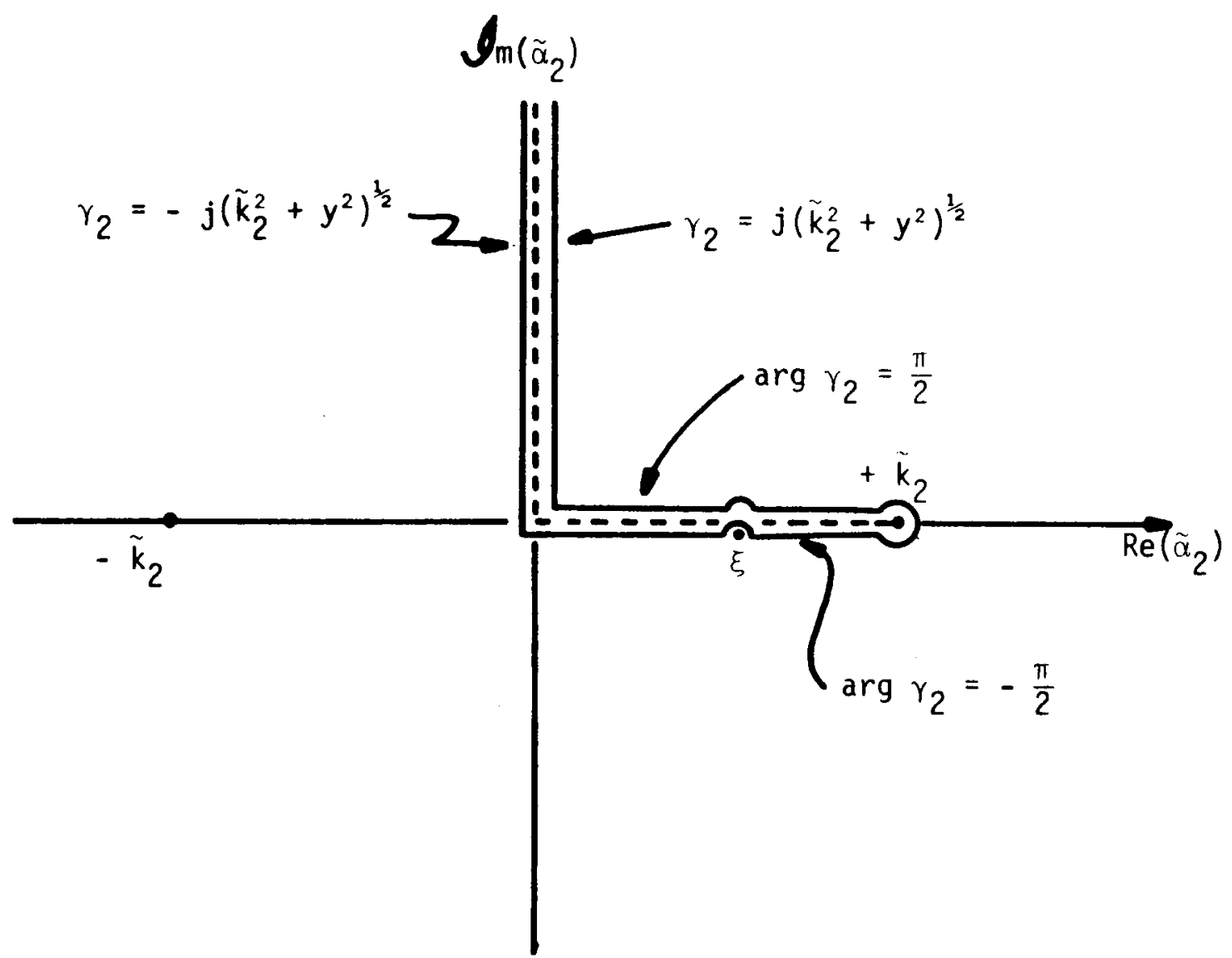

Figure 3 Path of integration. 


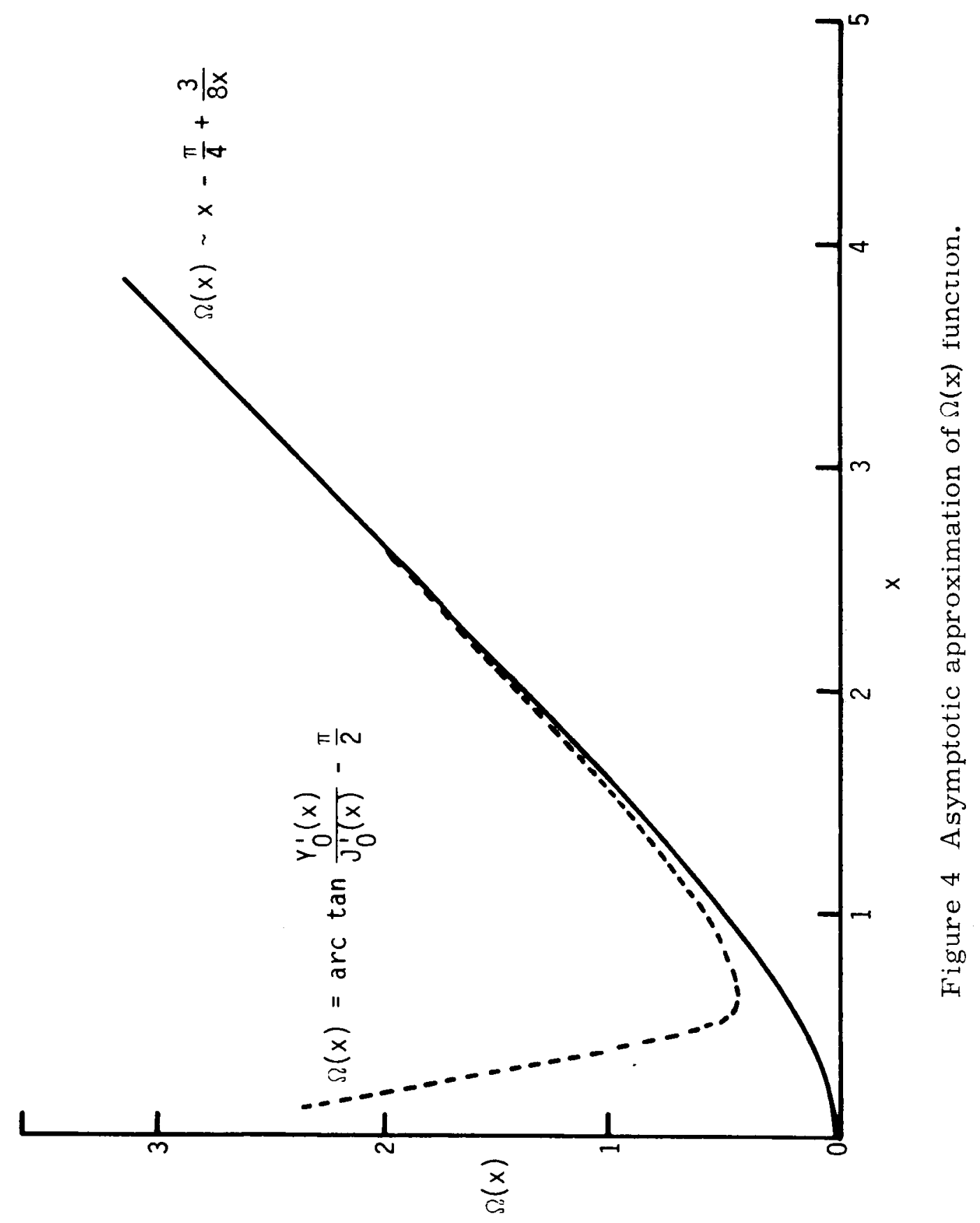




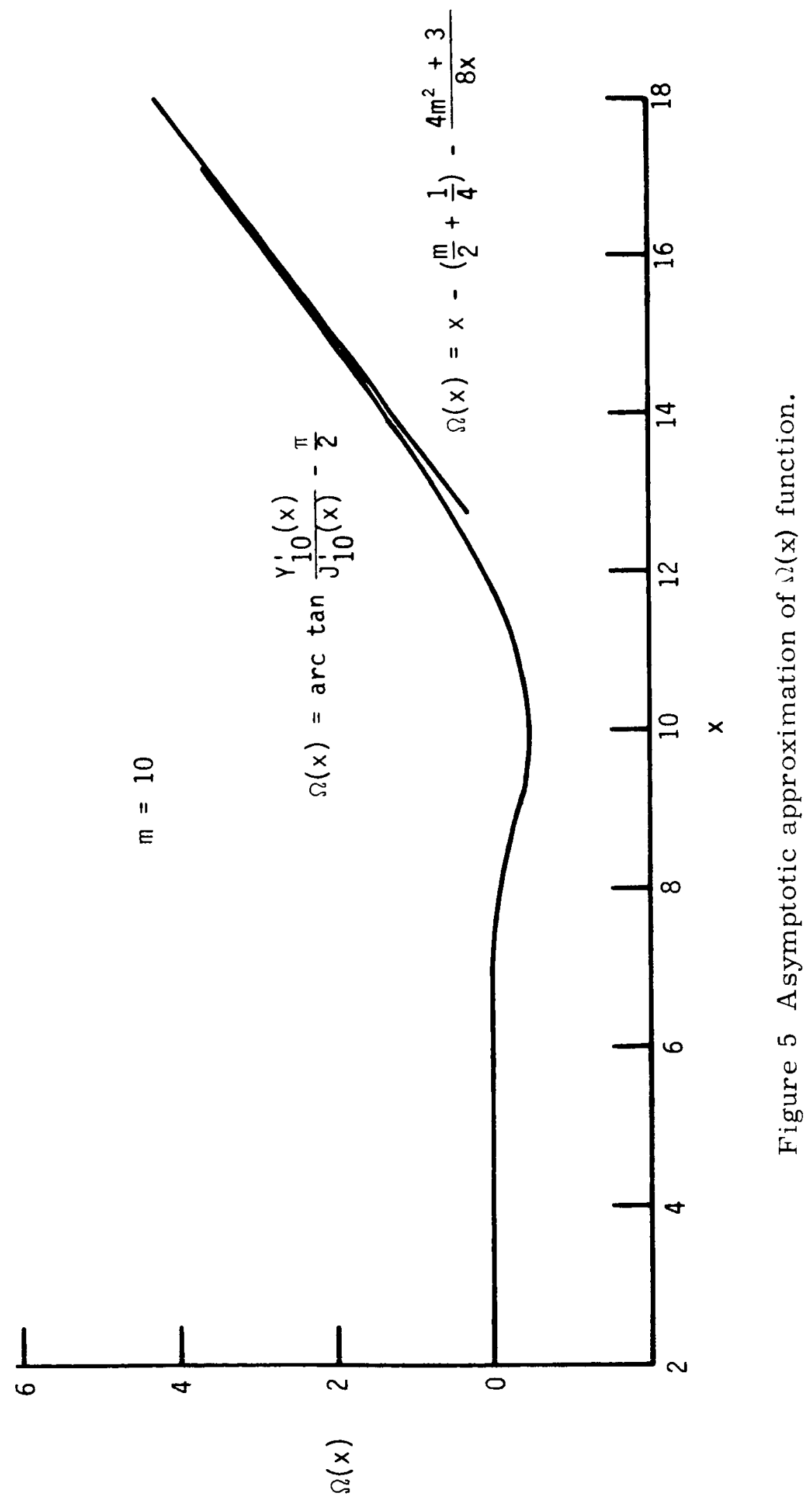




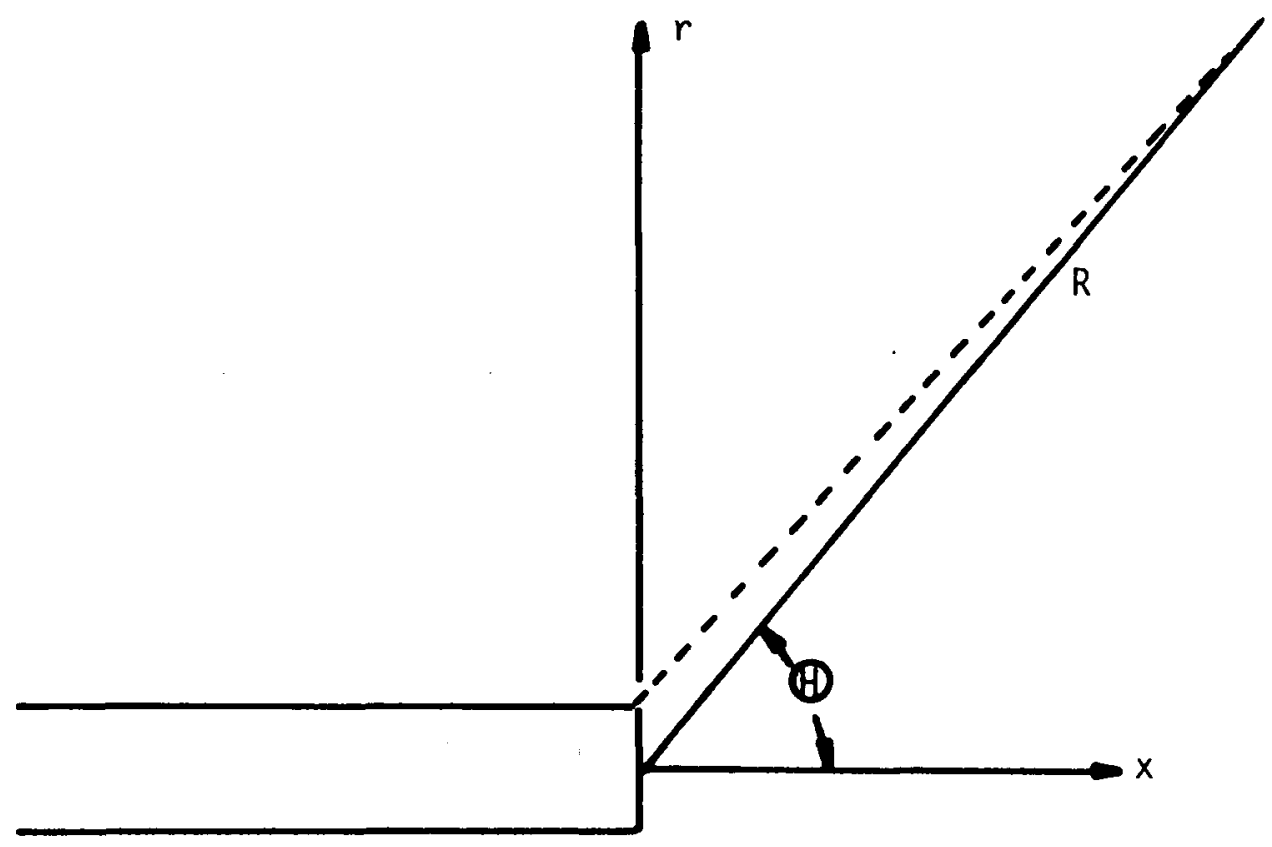

Figure 6 The radiation field. 


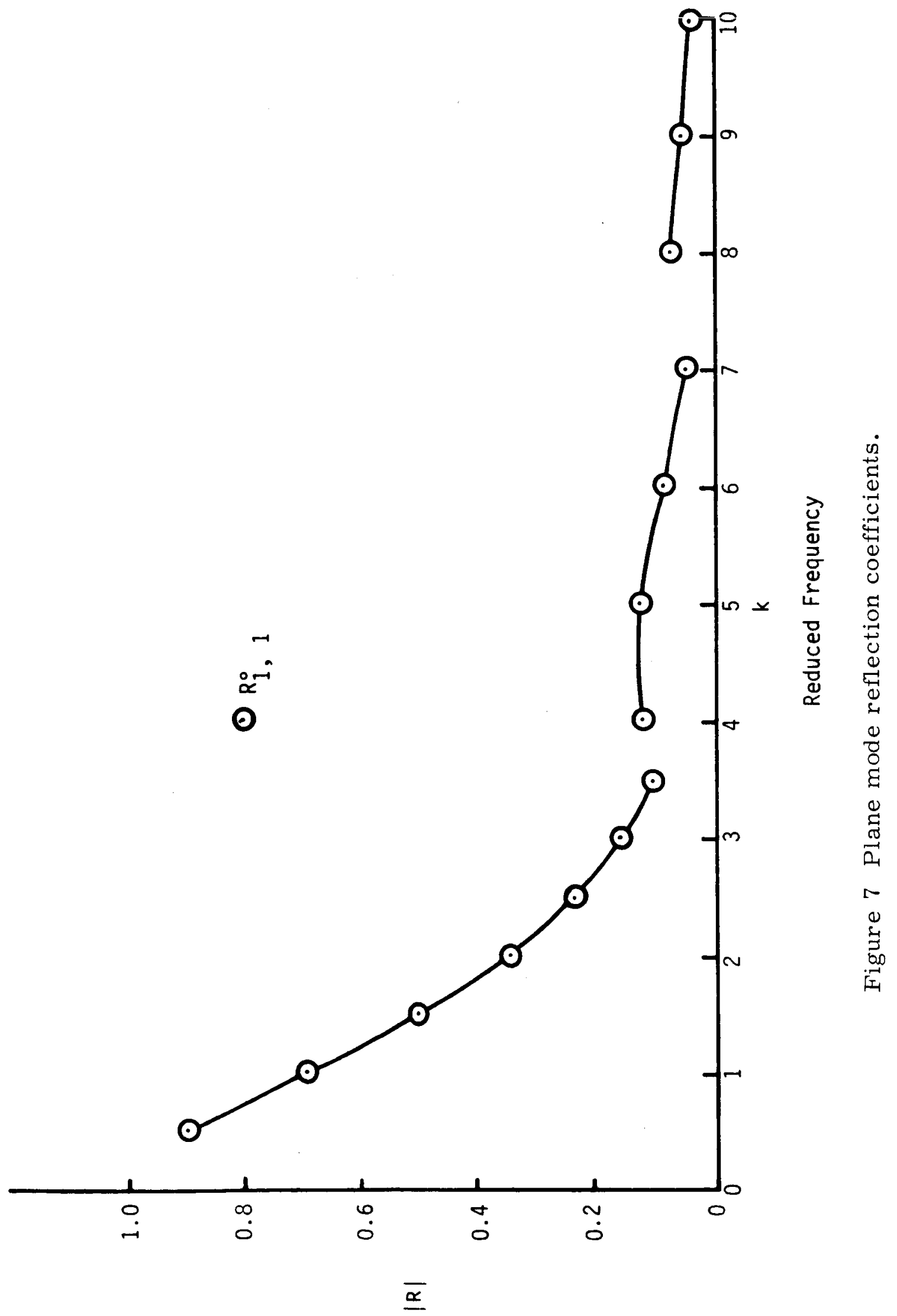




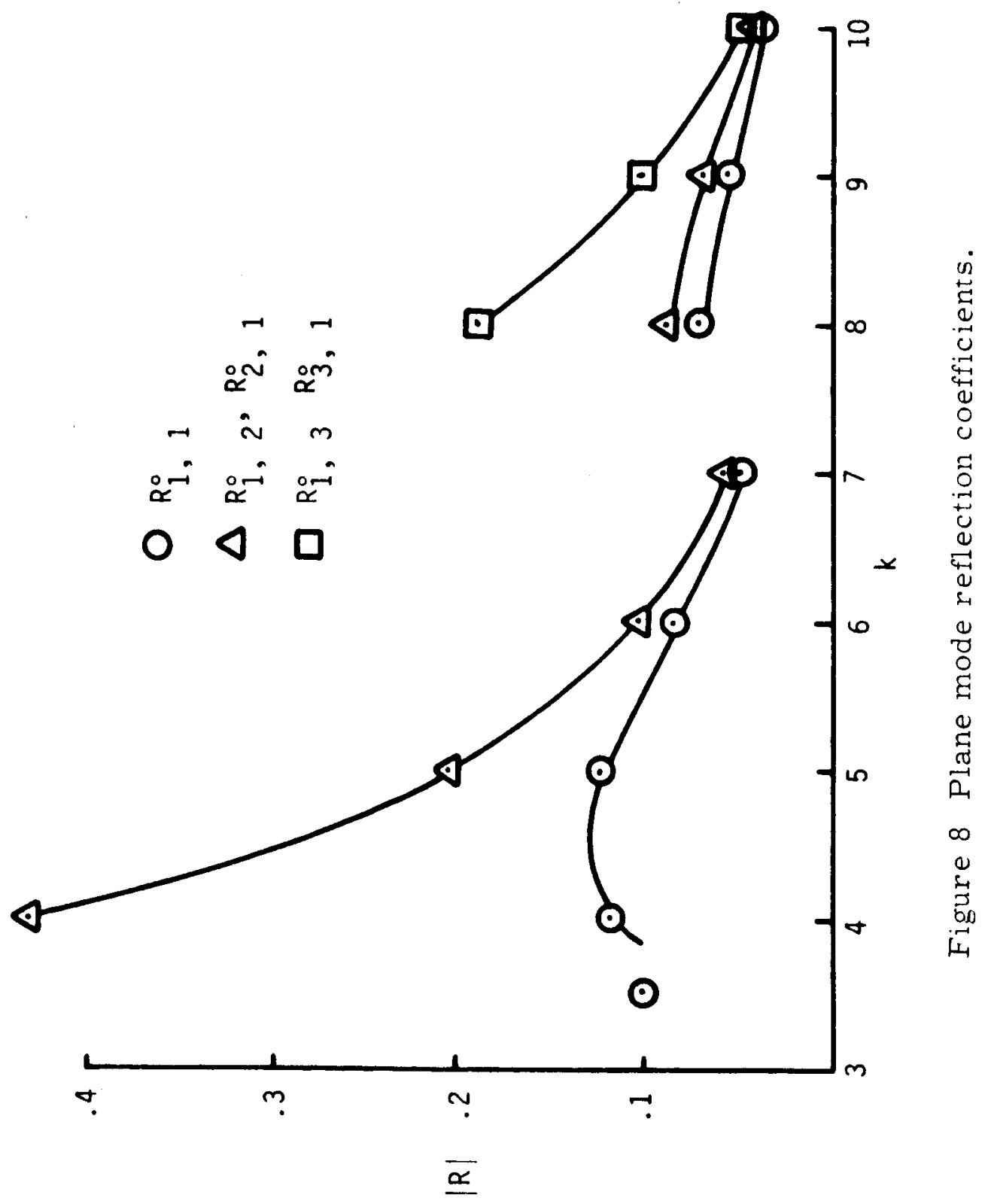




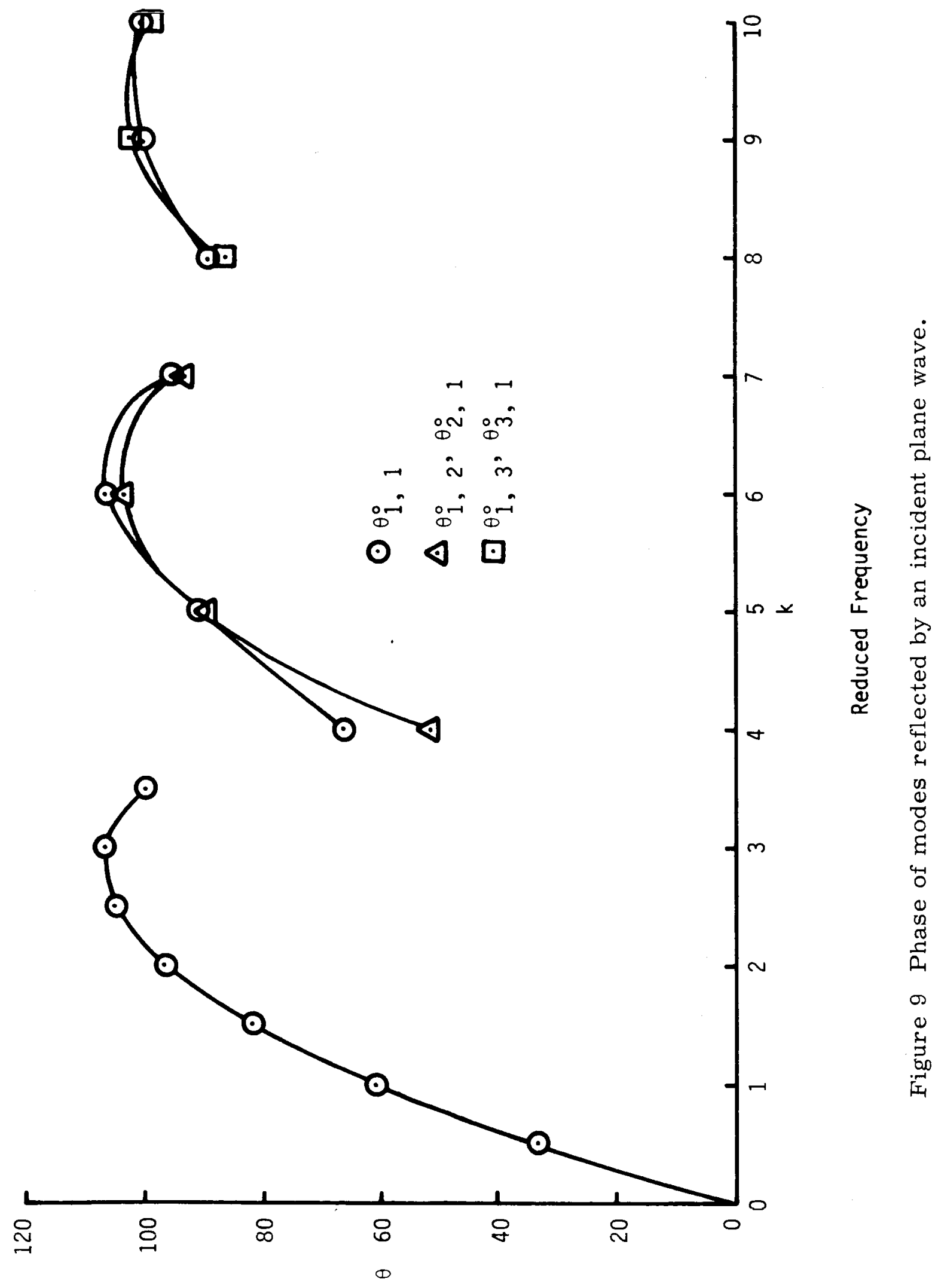

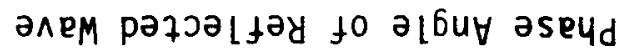




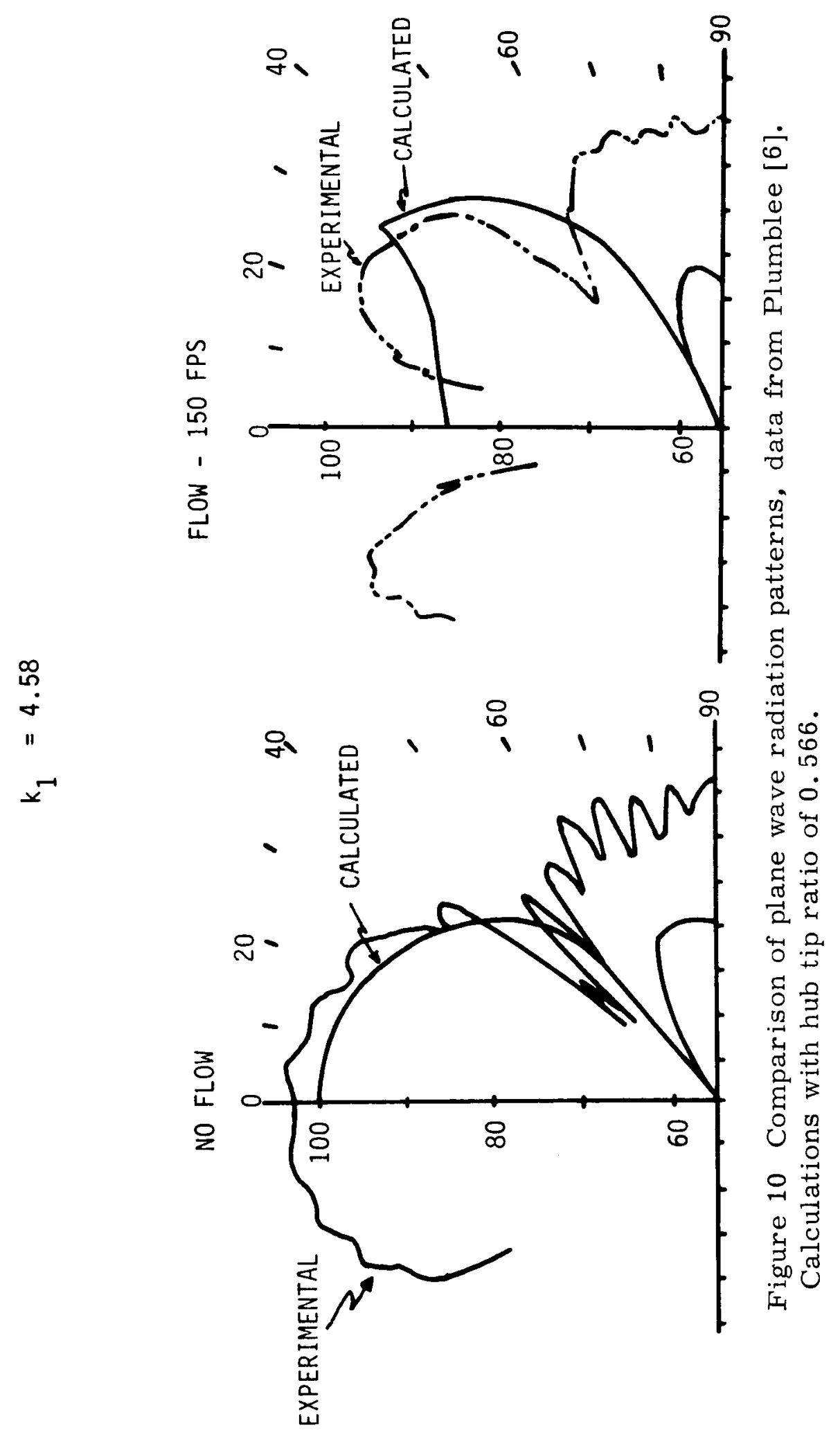




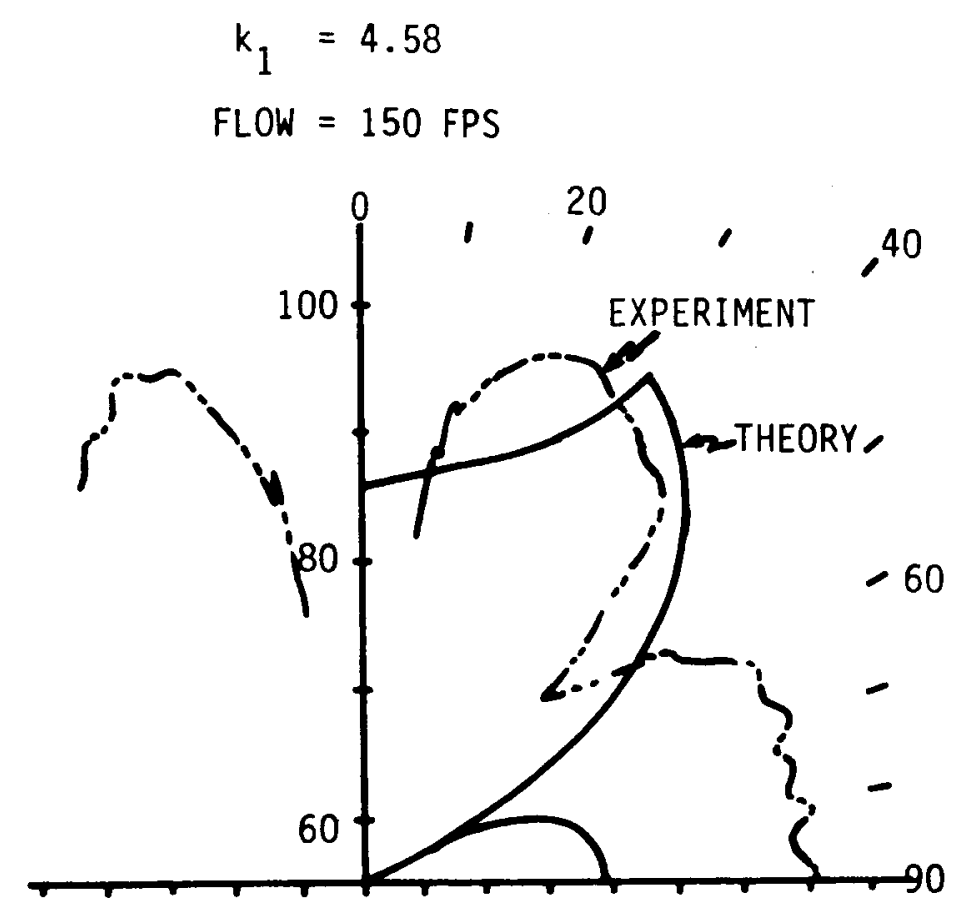

Figure 11 Comparison of a hubless plane wave radiation pattern with data of Plumblee [6]. 


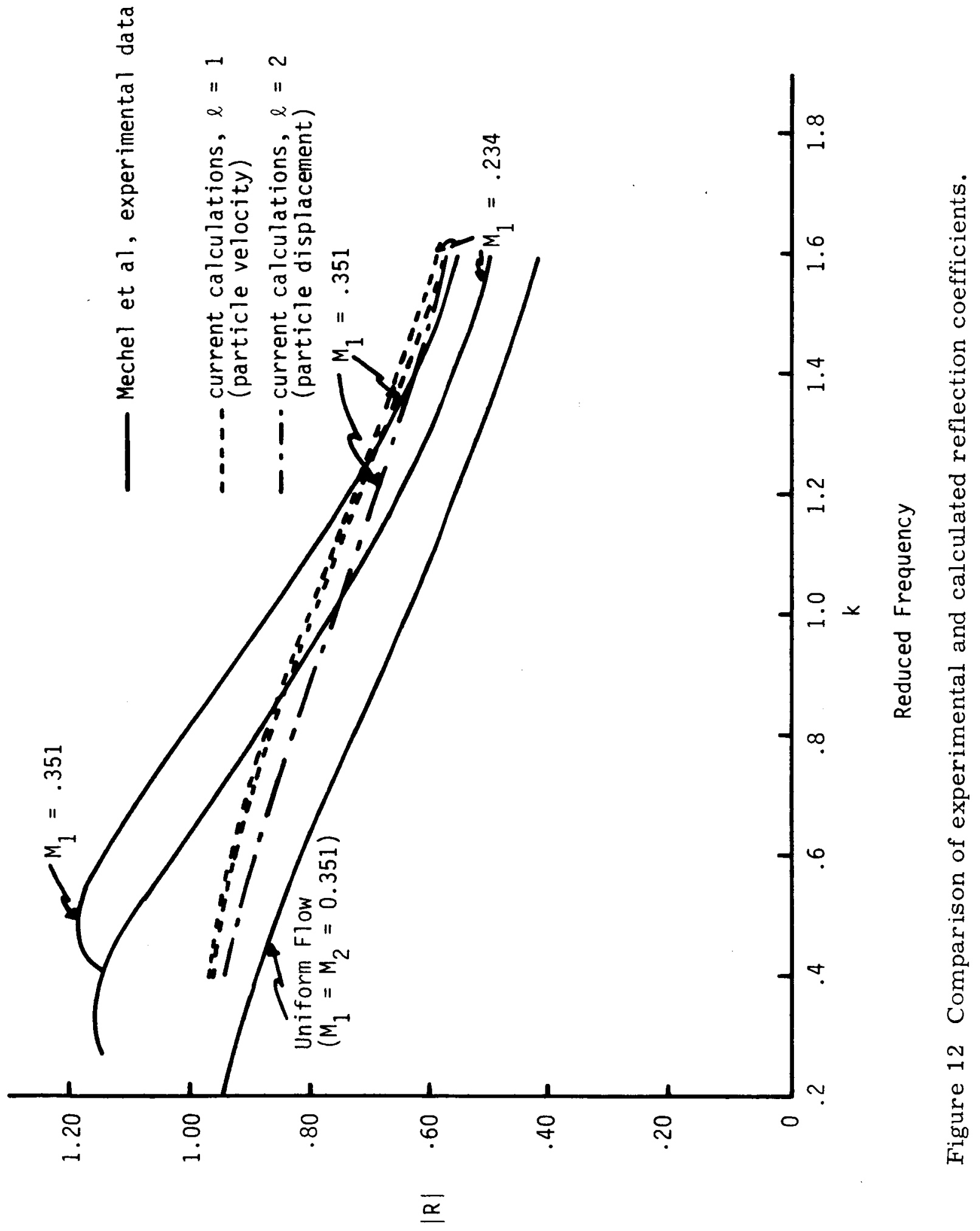


$k_{1}=.5$

PARTICLE DISPL.ACEMENT MATCH

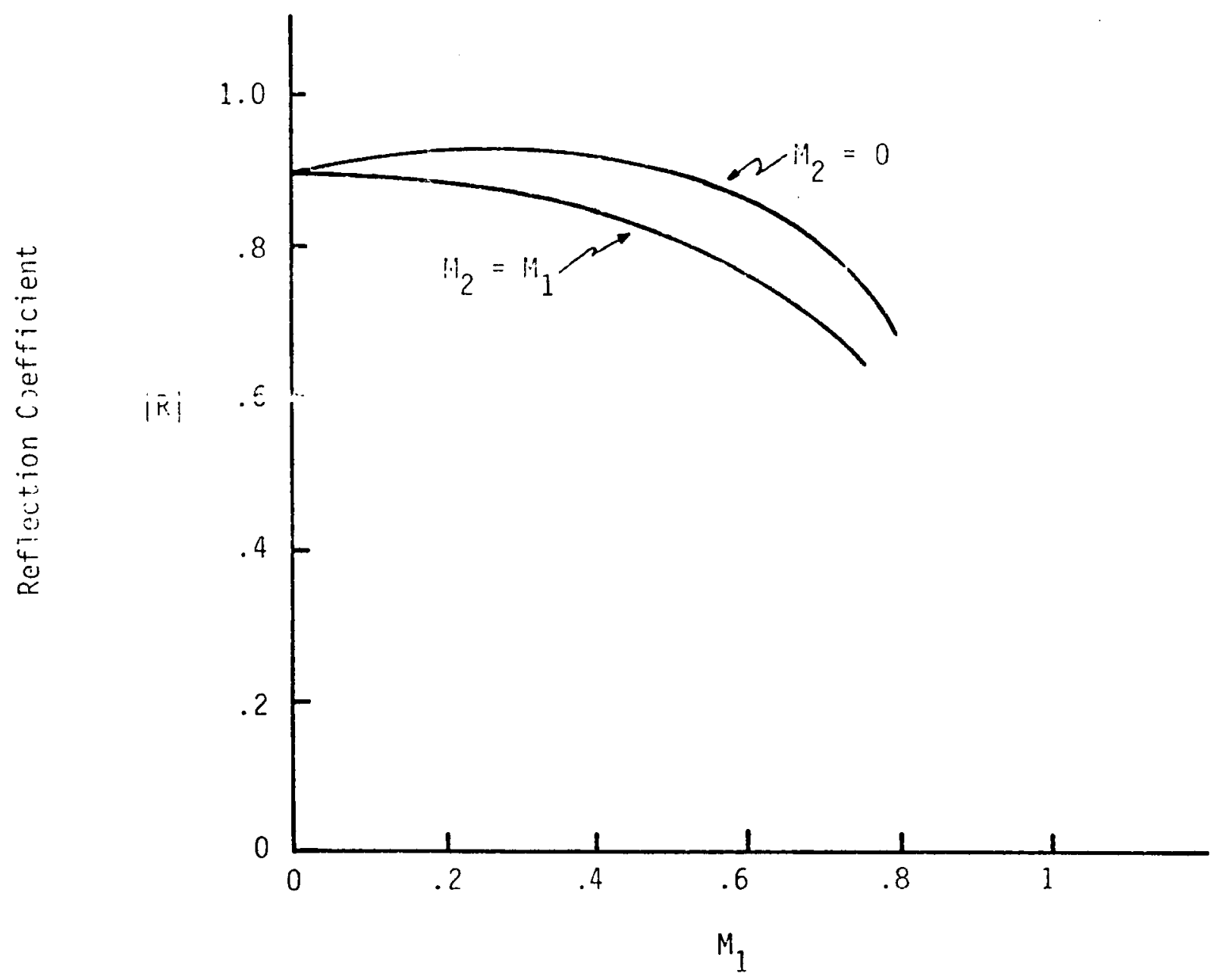

Figure 13 Calculated effect of Mach number on the plane wave reflection. 


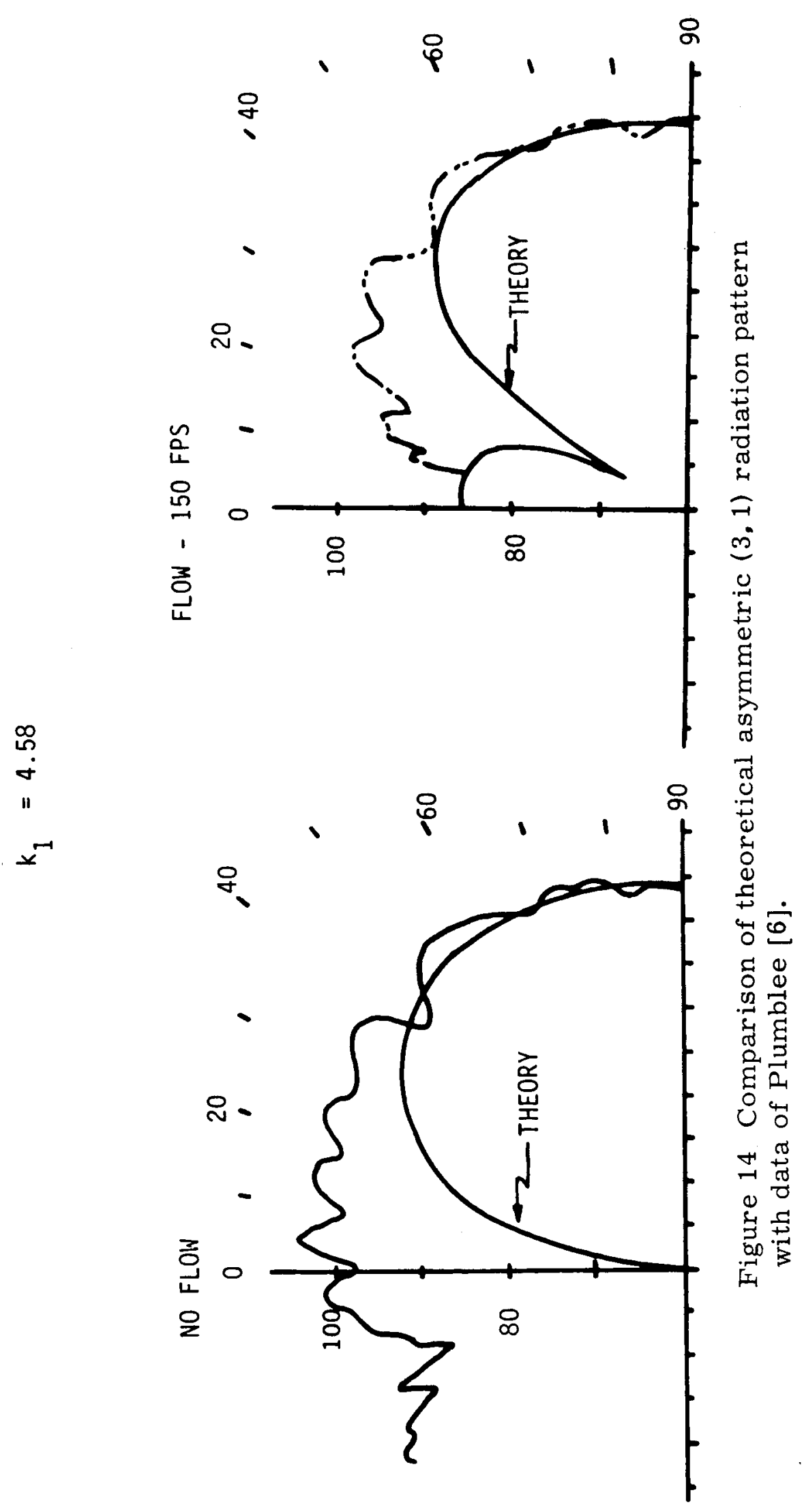


$m=4 \quad n=1 \quad k_{1}=11.7 \quad M_{1}=.14$

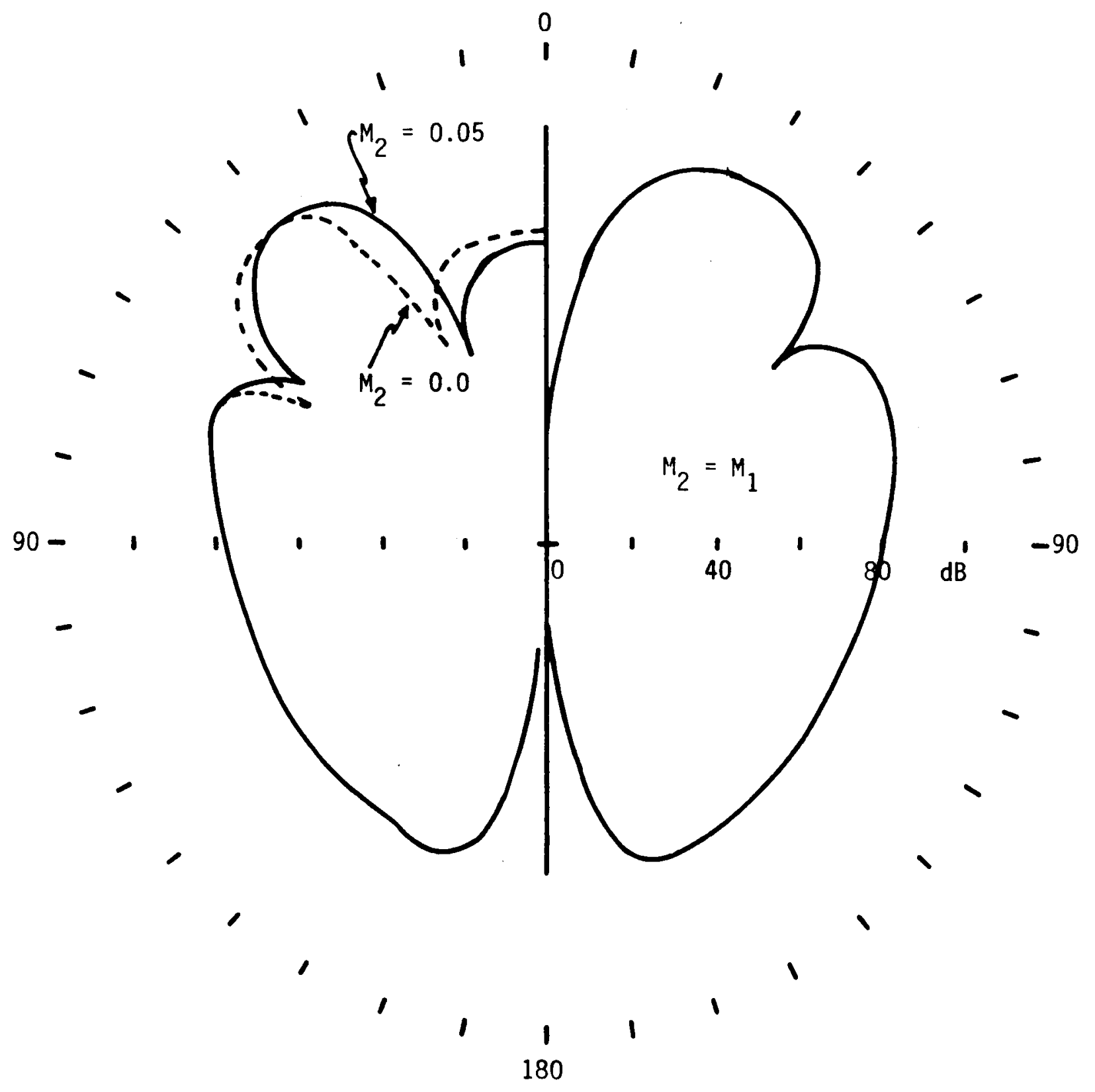

Figure 15 Illustration of the influence of flow mismatch and flight effect. Compare with data of Plumblee [6] for the $(4,1)$ mode. 
$m=10 \quad n=1 \quad k_{1}=15 \quad M_{2}=0$

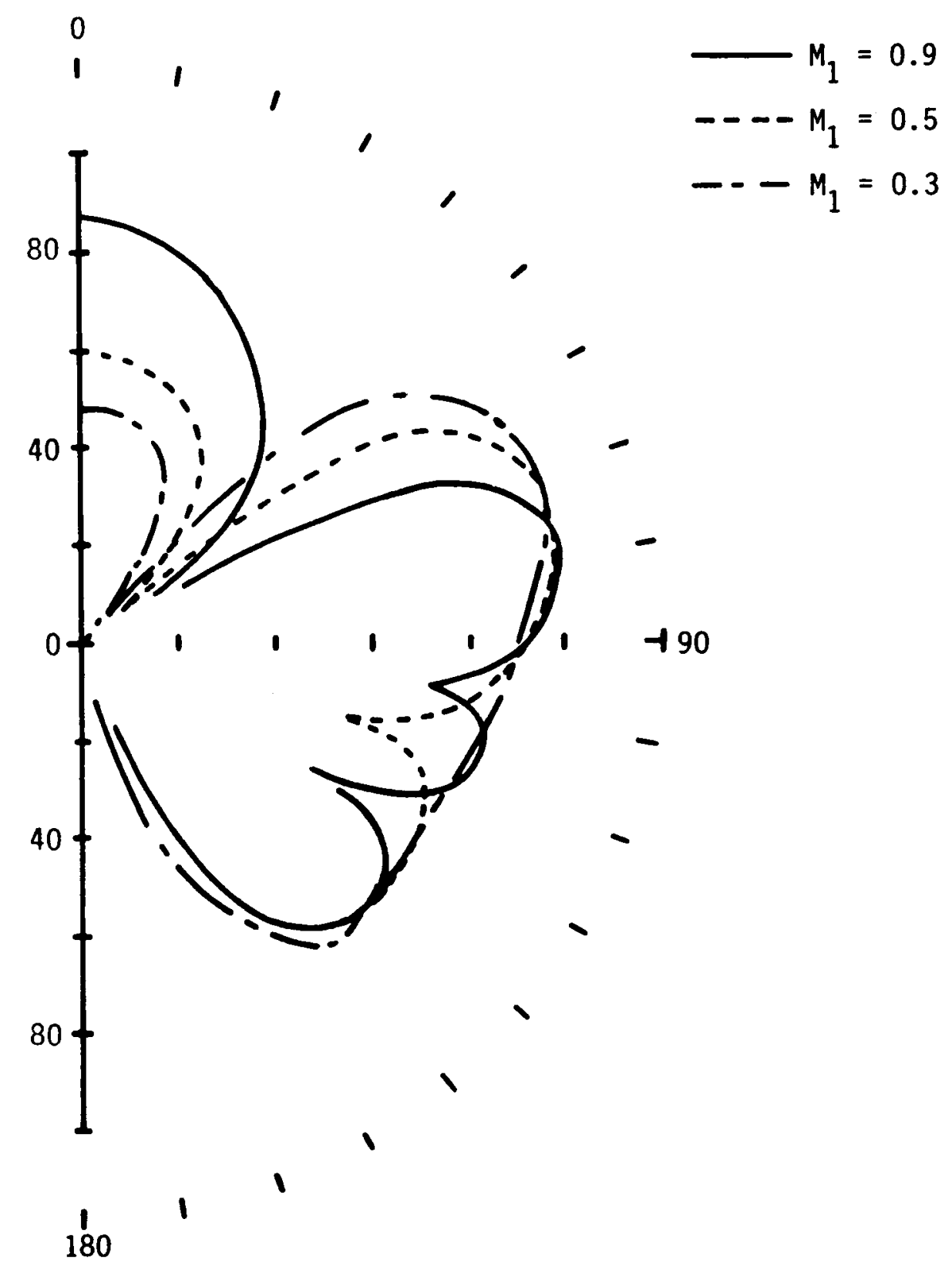

Figure 16 Effect of increasing jet issue Mach number. 


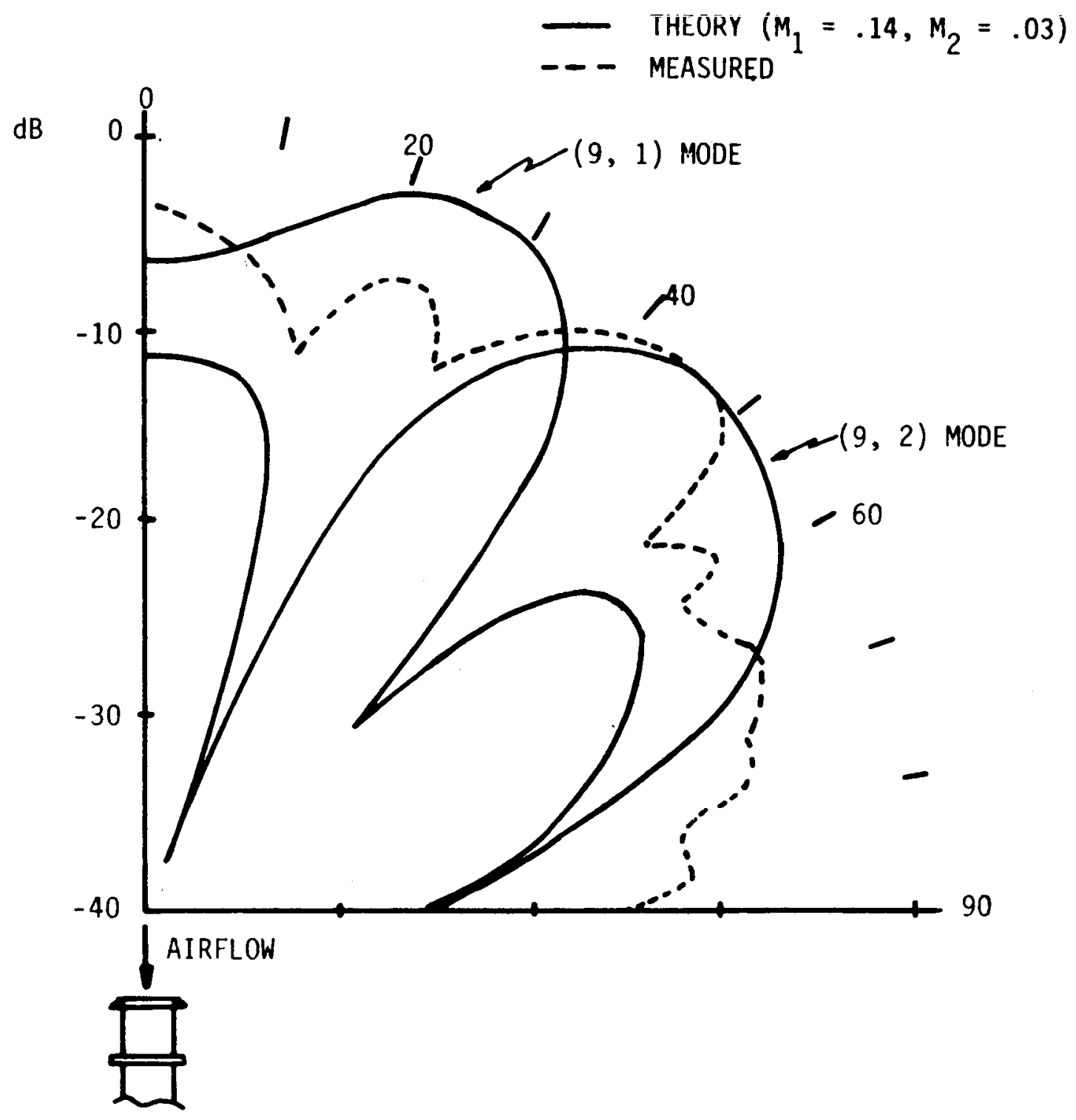

Figure 17 Trial calculations for an inlet mode and comparison with Crigler and Copeland [8] fan data for 53 bladed fan and 62 bladed IGV (9 lobe spinning mode). 


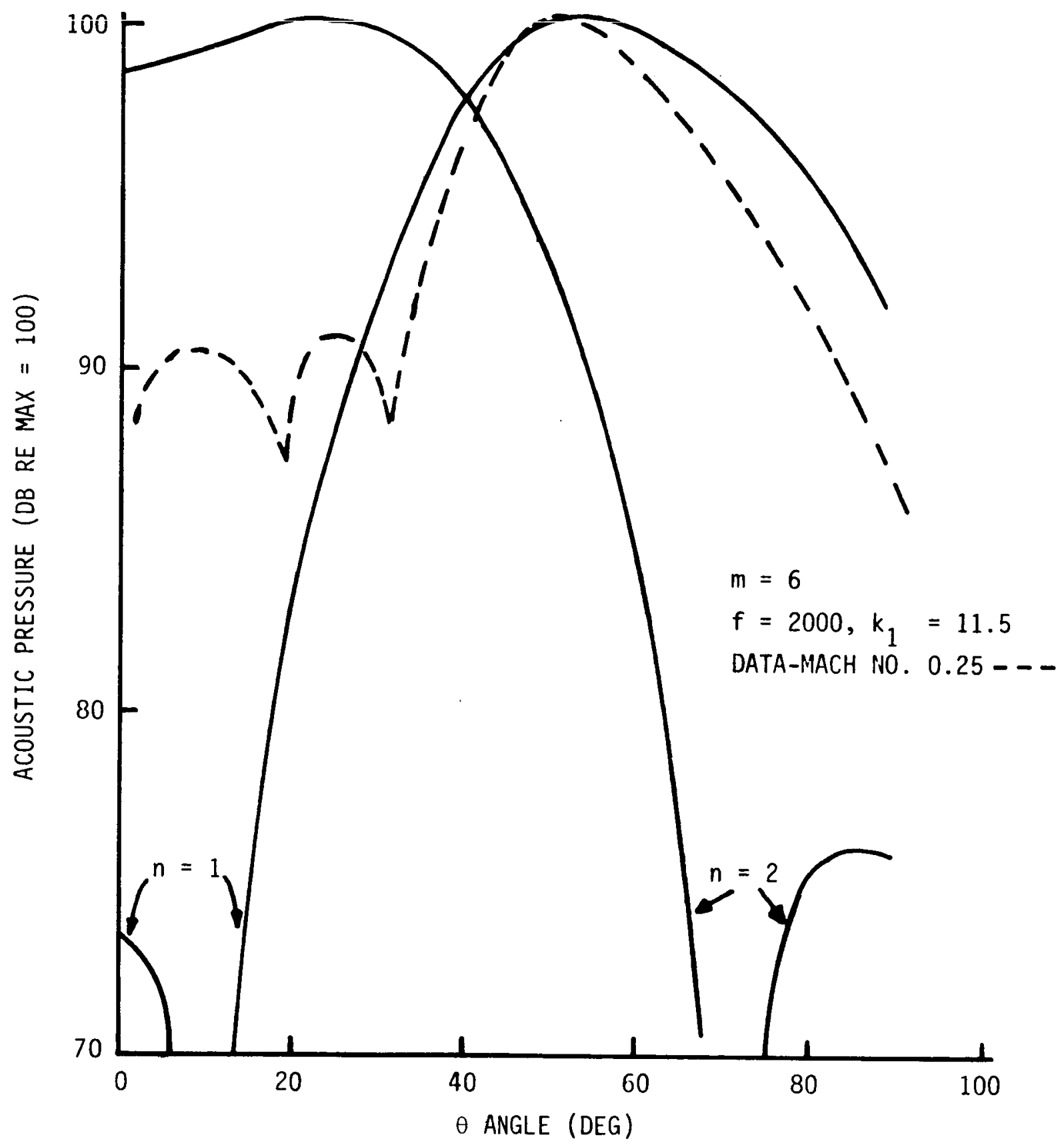

Figure 18 Annular inlet directivity patterns, comparison of theory with phase speaker array data of Pasko [19]. 
$m=4 \quad n=1 \quad M_{1}=.14 \quad k_{1}=11.7 \quad R C=C_{2} / C_{1}$

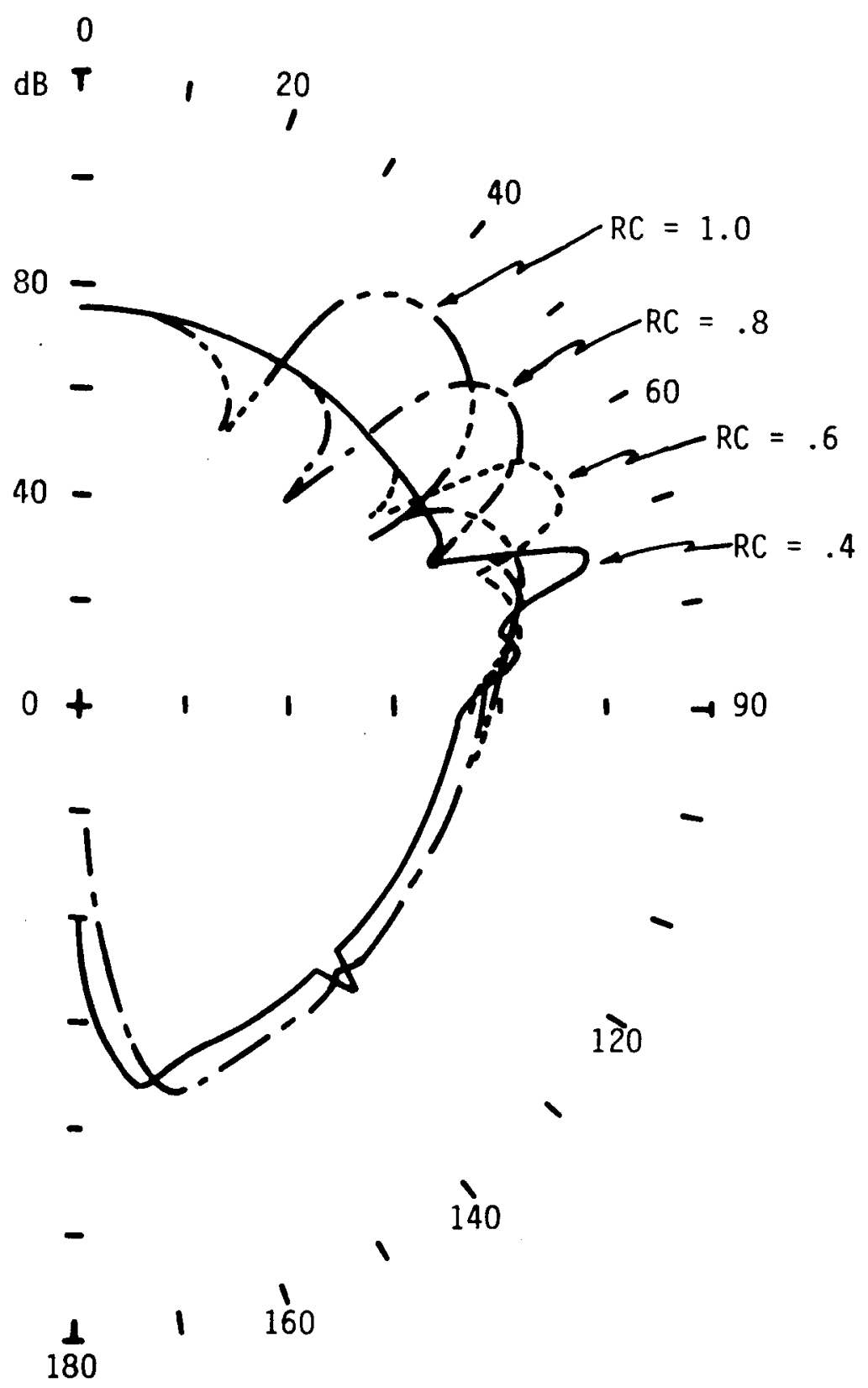

Figure 19 Effect of hot exhaust jet with fixed Mach number and reduced frequency. 
$m=4$

$\mathrm{n}=1$

$$
\mathrm{RC}=\mathrm{C}_{2} / \mathrm{C}_{1}
$$

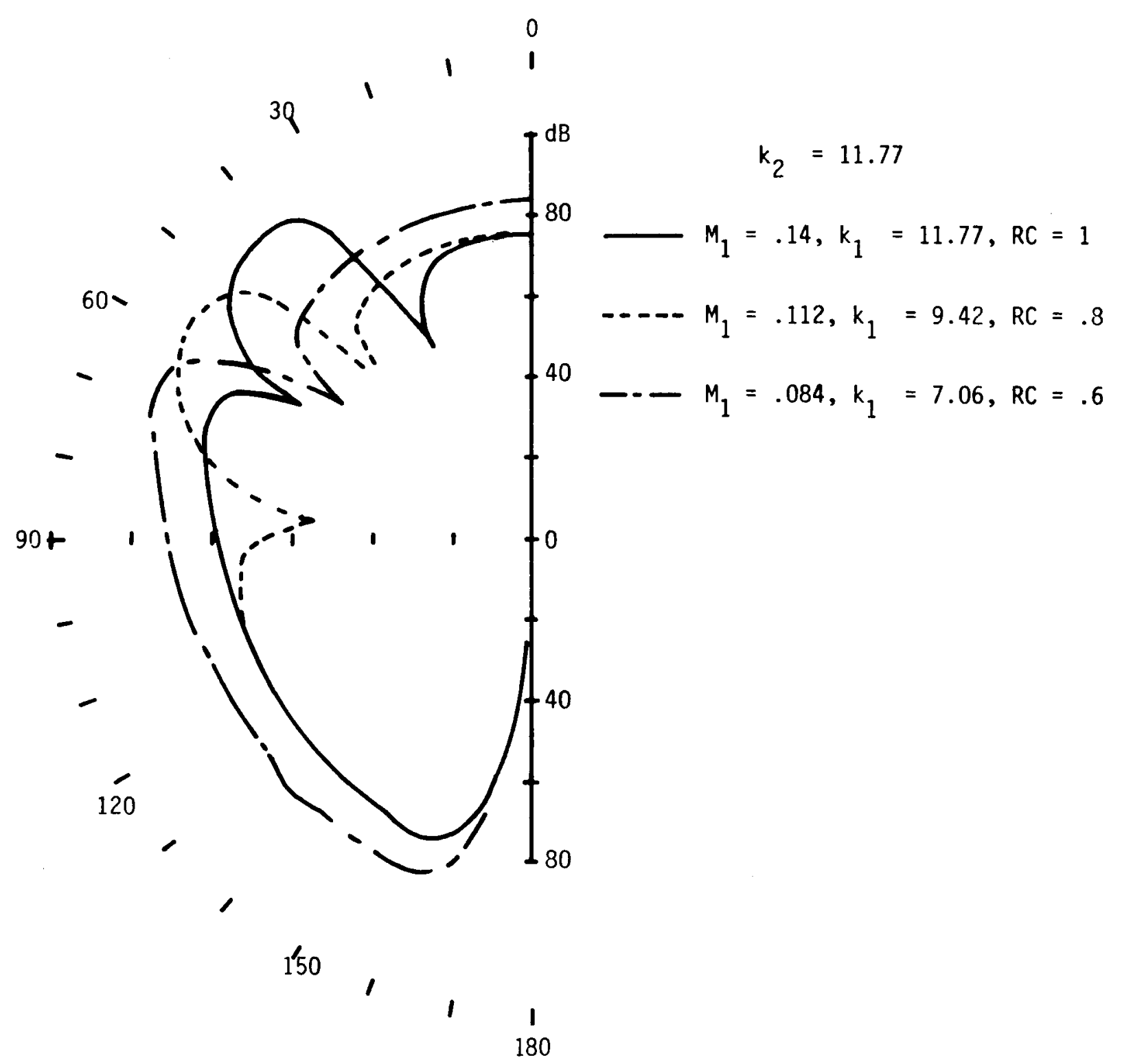

Figure 20 Effect of hot exhaust jet with fixed physical exhaust jet speed and sound frequency. 
NASA CR -132613

\section{DISTRIBUTION LIST}

NAS $1-11984$

Copies

NASA Langley Research Center

Hampton, VA 23665

Attn: Report \& Manuscript Control Office, Mail Stop 180A

Raymond L. Zavasky, Mail Stop 115

Dr. William E. Zorumski, Mail Stop 239

NASA Ames Research Center

Moffett Field, CA 9,4035

Attn: Library, Mail Stop 202-3

NASA Flight Research Center

P. O. Box 273

Edwards, CA 93523

Attn: Library

NASA Goddard Space Flight Center

Greenbelt, MD 20771

Attn: Library

NASA Johnson Space Center

2101 Webster Seabrook Road

Houston, TX 77058

Attn: JM6/Library

Jet Propulsion Laboratory

4800 Oak Grove Drive

Pasadena, CA 91103

Attn: Library, Mail 111-113

NASA Lewis Research Center 21000 Brookpark Road

Cleveland, OH 44135

Attn: Library, Mail Stop 60-3

NASA John F. Kennedy Space Center

Kennedy Space Center, FL 32889

Attn: Library, IS-DOC-1L

NASA Marshall Space Flight Center

Huntsville, AL 35812

Attn: Library 
National Aeronautics \& Space Administration

Washington, DC 20546

Attn: KSS-10/Library

RL/NASA Headquarters

The Lockheed Aircraft Corporation

Lockheed-Georgia Company

86 South Cobb Drive

Marietta, GA 30060

Attn: John S. Gibson, Dept. 72-47, Zone 13

McDonnell Douglas Corporation

Douglas Aircraft Company

3855 Lakewood Boulevard

Long Beach, CA 90808

Attn: Robert E. Pendley

General Electric Company

Evendale, $\mathrm{OH} 45215$

Attn: Robert Lee, Acoustic Design Technology

Aircraft Engine Group, Mail Drop H-77

Boeing Vertol Company

P.O. Box 16858

Philadelphia, PA 19142

Attn: Harry Sternfeld, Mail Stop P38-36

Detroit Diesel Allison

P.O. Box 894

Indianapolis, IN 46206

Attn: Harry Drye, U29A

The Boeing Company

3801 South Oliver Road

Wichita, KS 67210

Attn: Henry Veldman, Mail Stop K90-20

The Boeing Company

Boeing Commercial Airplane Company

P. O. Box 3707

Seattle, WA 98124

Attn: Robert P. Gerend, Aircraft Noise Staff - Research Unit 
Lockheed Aircraft Corporation

Lockheed-California Company

P. O. Box 551

Burbank, CA 91520

Attn: Harry Drell, Department Manager for Acoustics

Department 74-15, Building 63, Plant A1

United Aircraft Corporation

Hamilton Standard

Bradley Field Road

Windsor Locks, CT 06118

Attn: F.B. Metzger

Pratt \& Whitney Aircraft Company

400 Main Street

East Hartford, CT 06118

Attn: J. Kester, EB1F

United Aircraft Corporation

Sikorsky Aircraft Company

Stratford, CT 06602

Attn: Charles L. Munch, Aeromechanics Branch Supervisor Acoustics Section

NASA Scientific \& Technical Information Facility

P. O. Box 33

College Park, MD 20740 